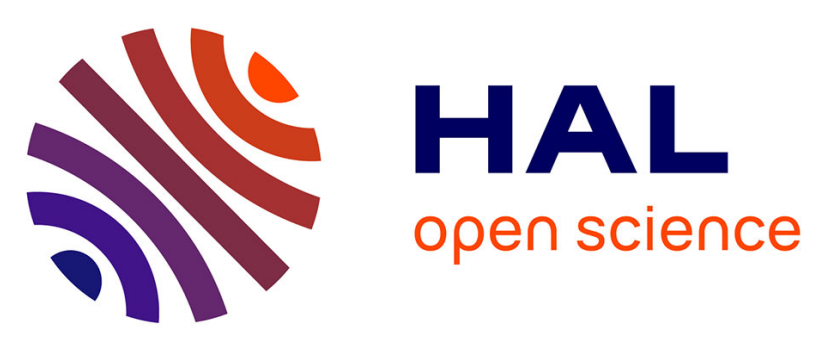

\title{
A study on the impacts of maintenance duration on dynamic grouping modeling and optimization of multicomponent systems
}

Hai-Canh Vu, Phuc Do, Anne Barros

\section{- To cite this version:}

Hai-Canh Vu, Phuc Do, Anne Barros. A study on the impacts of maintenance duration on dynamic grouping modeling and optimization of multicomponent systems. IEEE Transactions on Reliability, 2018, 67 (3), pp.1377-1392. 10.1109/TR.2018.2827926 . hal-02489046

\author{
HAL Id: hal-02489046 \\ https://hal.science/hal-02489046
}

Submitted on 24 Feb 2020

HAL is a multi-disciplinary open access archive for the deposit and dissemination of scientific research documents, whether they are published or not. The documents may come from teaching and research institutions in France or abroad, or from public or private research centers.
L'archive ouverte pluridisciplinaire HAL, est destinée au dépôt et à la diffusion de documents scientifiques de niveau recherche, publiés ou non, émanant des établissements d'enseignement et de recherche français ou étrangers, des laboratoires publics ou privés. 


\title{
A study on impacts of maintenance duration on dynamic grouping modeling and optimization of multi-component systems
}

\author{
Hai-Canh $\mathrm{Vu}^{1}$, Phuc Do ${ }^{1 *}$ Anne Barros ${ }^{2}$ \\ ${ }^{1}$ Université de Lorraine, CRAN, UMR 7039, Campus Sciences, BP 70239, Vandoeuvre-les-Nancy, 54506, France \\ ${ }^{2}$ Department of Mechanical and Industrial Engineering, Norwegian University of Science and Technology, Trondheim, Norway
}

\begin{abstract}
In the framework of maintenance optimization of multi-component systems, dynamic grouping maintenance based on rolling horizon has developed and becomes an interesting approach. However, most existing dynamic grouping models assume that the maintenance duration is negligible. This assumption may be not always relevant and limits the application of these models in many real situations. The first objective of this paper is to develop a dynamic grouping model in presence of both corrective and preventive maintenance duration for complex structure systems. A new practical maintenance cost model is proposed allowing to take into consideration of maintenance duration and system structure as well as economic dependence between components. Taking into maintenance duration and system structure leads however to a complex grouping model which is efficiently solved by proposed analytical methods. The second objective is to investigate the impacts of maintenance duration on grouping modeling and grouping optimization. Both theoretical and experimental studies give a complete vision about maintenance duration impacts and some recommendations in real applications. The uses and advantages of the proposed grouping maintenance approach are illustrated through a numerical example of a six-component system.
\end{abstract}

Keywords: Maintenance modeling, maintenance optimization, dynamic grouping, multi-component system, maintenance duration.

\section{Introduction}

Maintenance optimization nowadays plays a key role in ensuring system functions at lower cost, failing to do so will expose the system owner to very large costs. With fast economic growth and developing advanced technologies, manufacturing systems become more and more complex. They involve a high number of components where maintenance is necessary to maintain their performance throughout the lifetime. In this context, investigations on maintenance optimization tend to change from mono-component models (see, for instance, $[1,2,3,4])$ towards multi-component ones. As underlined by a number of review papers $[5,6,7,8]$, the multicomponent maintenance optimization models is more accurate than mono-component ones, since they allow to

${ }^{*}$ Corresponding Author: phuc.do@univ-lorraine.fr 
take into account components' dependencies. The dependencies among components are usually classified into three different kinds: economic, stochastic and structural dependence $[9,10,11,12]$, in which the economic dependence has been most studied and integrated in maintenance optimization process. When components are economically dependent, the cost of joint maintenance of these components is not equal to the total cost of their separate maintenance. Indeed, the joint maintenance can lead to a higher maintenance cost (negative economic dependence) or a lower maintenance cost (positive economic dependence) when compared to the case where the components are separately maintained $[13,14]$.

To take advantages of the positive economic dependence, opportunistic and grouping maintenance approaches have been proposed. The main idea is to jointly maintain several maintenance activities to reduce the maintenance cost. The opportunistic maintenance focuses on doing the preventive maintenance (PM) of several components at maintenance opportunities such as system downtimes due to failures, production plan or safety constraints. This approach has been extensively developed in a number of papers and successfully applied to the maintenance planning of industrial systems such as oil and gas, electrical distribution $[15,16,17,18]$. However, the maintenance opportunities such as system failures occur randomly, the opportunistic maintenance plan is then not fixed in advance, and brings a lot of difficulties to the logistic supports and maintenance management. These difficulties limit the applications of the opportunistic maintenance in real situations. In this paper, we focus on the second approach: grouping maintenance.

The grouping maintenance is intent to reduce the maintenance cost and improve the system performance (system's reliability, system's availability) by jointly performing several PM activities. A number of papers working on this topic have been recorded in the literature and can be classified into three different types according to the planning horizon: short-term horizon, long-term horizon, and rolling horizon. The grouping maintenance within a short-term planning horizon [19, 20, 21] does not guarantee the grouping performance within long-term horizon. While the grouping maintenance within long-term planning horizon [22, 23] does not allow to dynamically update the maintenance planning. The grouping maintenance based on rolling horizon therefore has received a lot of attention from the research community since it holds the advantages of both the short-term and long-term maintenance planning.

The dynamic grouping based on rolling horizon has been firstly introduced in [24] for series systems. The approach starts by finding an optimal PM cycle for each component which minimizes its long-term maintenance cost rate. The tentative PM activities are then determined within a short-term horizon and optimally grouped to save setup cost. The approach has been then developed to take into account: the component degradation information in [25], the prognostic and/or diagnostic information in [26], the human factors in [27, 28], the stochastic dependence in [29], the dynamic contexts (maintenance opportunities) and maintenance constraints such as availability constraint, repairman constraint in [30, 31, 32], the complex structure (which can be any combination of basic structures such as series, parallel, or k-out-of-n) in [30, 13].

Most of the above works rely on the assumption that the corrective maintenance (CM) duration and/or PM duration can be neglected to simplify the maintenance modeling and maintenance optimization at both component and system levels (see Table 1 for more details). However, this assumption may lead to sub-optimal grouping solution. In addition, since a number of grouping maintenance calculus are done in finite horizons, the maintenance duration takes a significant part when compared to the horizons' length and can not be neglected. For these above reasons, some recently works have taken efforts to overcome this problem. Indeed, PM duration 
Table 1: Dynamic grouping maintenance approaches based on rolling horizon

\begin{tabular}{lllc}
\hline Papers & Maintenance duration & System structure & $\begin{array}{c}\text { Methods for grouping's } \\
\text { performance assessment }\end{array}$ \\
\hline \hline$[24,27]$ & No & Series & Analytic \\
{$[25]$} & No & Series & Simulation \\
{$[29]$} & No & Parallel & Simulation \\
{$[30]$} & No & Complex & Analytic \\
{$[31,32]$} & PM duration & Series & Analytic \\
{$[28]$} & PM duration & Series \& Parallel & Analytic \\
{$[13]$} & PM duration & Complex & Analytic \\
{$[26]$} & PM \& CM duration & Series & Simulation \\
\hline \hline
\end{tabular}

is considered and integrated into grouping optimization process in papers [31, 32, 28, 13]. The consideration of the PM duration is quite simple when compared to that of the CM duration since the PM is usually planned at determined dates. Given the randomness of failures, the CM carried out at failures is unknown in advance. The taking into account of CM duration is a real challenge, especially in case of complex structures. Paper [26] overcomes the problem by proposing a Monte Carlo simulation process. The use of the simulation process requires a huge computational resources and limits the proposed methods in online updating and in some real applications. Nevertheless, the maintenance approach proposed in [26] can be applied only for series systems.

The first objective of this paper is to develop a grouping maintenance approach taking into account both corrective and preventive maintenance duration for multi-component systems with complex structure. Moreover, to our best knowledge, there is a lack of deep and complete analysis about the impacts of maintenance duration on grouping modeling and grouping optimization. The second objective of the paper is then to carry out theoretical and experimental analysis on these impacts. To achieve these two objectives,the following main contributions are provided in this paper:

- Proposition of a new practical maintenance cost model allowing to take into consideration of maintenance duration and system structure as well as economic dependence between components. The cost model is especially suitable for the maintenance modeling of complex structure systems.

- Develop a new dynamic grouping process for the maintenance of complex structure systems with nonnegligible maintenance duration by introducing two analytical methods which llow to track the changes of system structure and their components' age over time. The proposed analytical process can be used for online maintenance decision-making, and helps to improve the grouping maintenance performance in real applications.

- Analyze impacts of maintenance duration on the maintenance modeling and maintenance optimization at both component level and system level. The analysis results give a complete vision about maintenance duration impacts and some recommendations in real applications.

The rest of the paper is organized as follows. Section 2 is devoted to present the considered system, some general assumptions, and the new maintenance cost model. The grouping maintenance approach is described in Section 3. Moreover, the impacts of maintenance duration in the grouping modeling and grouping optimization 
are herein analyzed. analytical methods for evaluating components criticality and age which are used in the grouping process are developed in Section 4. In Section 5, numerical analysis are carried out for a complex structure system composed of 6 components to study the impacts of maintenance duration, and to show how the developed grouping approach can be used. Finally, the last section presents some conclusions drawn from this work.

\section{Problem descriptions and general assumptions}

This section focuses on positioning the considered problem by starting with some general descriptions of multicomponent systems and its maintenance activities. The difficulties in maintenance modeling and maintenance optimization for such systems when the maintenance duration is taken into account are then identified.

\subsection{System description}

Consider a multi-component system consisting of $n$ binary components, i.e. a component state is either "operational" or "failed". To accomplish a specific function, the system's components are inter-connected according to a redundant structure in term of reliability block diagram. Due to the complexity of the system structure, two kinds of components are here considered: a component is a critical one at $t$, if its non-functioning state at this instant directly results in a complete shutdown of the system; a component is said to be redundant (or non-critical) one at instant $t$ if the system can be still functioning when the component stops. As an illustrative example, a 5-component system with redundancy is shown in Figure 1. For the considered structure, component 1 is a critical one and the others are redundant.

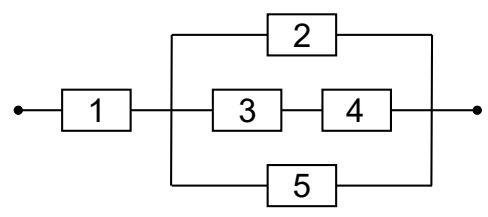

Figure 1: A redundant structure containing 5 components

According to the classification above, two basic structures are specified: (a) series structure: all components of the structure are critical for the system functioning. The non-functioning state of one component is enough to cause a complete shutdown of the system; (b) parallel structure: all components are redundant, i.e., in a normal operation state, a failure/stoppage of any component does not lead to the system shutdown.

Components of the system are subjected to random failures. To not lose sight of our main objective by considering too broad failure models, some following assumptions were considered: (a) component does not degraded when it does not work; (b) components are stochastically independent, i.e. the failure behavior of a component does not depend on that of the others; (c) probability of simultaneous failures is small and can be neglected; (d) the failure behavior of components is described by a continuous distribution with the increasing failure rate. To facilitate the understanding of the developments, Weibull distribution law is used as an example to describe the failure behaviors of the components. In that way, the failure rate of component $i(i=1, \ldots, n)$ 
at time $t$, denoted $z_{i}(t)$, is described by the following equation:

$$
z_{i}(t)=\frac{\beta_{i}}{\lambda_{i}}\left(\frac{x_{i}}{\lambda_{i}}\right)^{\beta_{i}-1}
$$

where, $\lambda_{i}>0$ and $\beta_{i}\left(\beta_{i}>1\right)$ are the scale and shape parameters of the Weibull distribution respectively. $x_{i}$ is the total operational time (the age) of component $i$ until time $t$. It should be noted that $x_{i}(t)=t$ only when the maintenance duration is neglected and component $i$ is put in operation at time $t=0$.

\subsection{Maintenance activities and cost structure}

Maintenance activities Given the diversity of maintenance activities within the system life cycle, a maintenance action can be classified in either preventive maintenance (PM) or corrective maintenance (CM). While preventive maintenance is carried out on functioning components in order to improve their health conditions and prevent them from an intensive regime of failures, corrective maintenance is executed on failed components to bring them to their operational state. In the paper, the preventive maintenance is considered to be able to restore the components to the "as good as new" state. Otherwise, after a corrective maintenance, the repaired components are in the state that they had just before their failure, in other words, the "as bad as old" state.

In real applications, for a such maintenance action (preventive or corrective one), it always takes time in executing. In general, the maintenance duration can contain the time for spare part ordering and transportation, machine opening, repairing, etc, and could not be neglected in some cases [5]. The maintenance duration is usually modeled as a fixed or stochastic amount of time [33, 31]. In the further analysis, the maintenance duration is considered to be a fixed amount of time and noted by $\omega^{i p}$ for PM duration and $\omega^{i c}$ for $\mathrm{CM}$ duration of component $i$ respectively. Moreover, since the $\mathrm{CM}$ is always directly associated to the component failure and its reparation, the two notations "CM duration" and "repair duration" are used interchangeably.

Cost structure This is an important issue in maintenance modeling, which has direct impacts on the performance of maintenance optimization and planning. A more general and complete maintenance cost structure is herein proposed, in which the total cost of a maintenance action on component $i$ is divided into five parts:

- Setup cost, denoted by $C_{s e}^{i-}$ with $-=p$ for preventive maintenance and $-=c$ for corrective maintenance, is paid for all preparation activities that allow the maintenance can be performed on component/system. The preparation activities could be, e.g, scaffolding erecting, machine opening, maintenance tools, or also traveling of maintenance teams, transportation of maintenance tools and spare parts. This setup cost can be shared when several components are jointly maintained [5, 31].

- Specific cost $\left(C_{s p}^{i-}\right)$ is the cost paid for spare part and depends on the specific characteristics of the component/system which is under maintenance.

- Labor cost is the cost paid for repairman for their maintenance work. Normally, this cost depends on the maintenance duration, the technician skill level, and is calculated through the average labor cost per time unit denoted by $c_{l a}^{i-}$.

- Shutdown cost has to be paid whenever the component/system fails or is stopped due to maintenance. This cost is associated with loss of quality of product or service, costs of restarting the component/system. Note that these costs may appear even when the component/system downtime is neglected. Let $C_{s h}^{i-}, C_{s h}^{s-}$ be the component shutdown cost and the system shutdown cost. 
- Downtime cost is paid for negative impacts of a period of time in which the component/system does not work. This downtime cost is associated with the unavailability of services or loss of products. The cost depends on the maintenance duration and is calculated through the average downtime cost rate. Two kinds of downtime cost rate are considered: component downtime cost rate $\left(c_{d o}^{i-}\right)$ and system downtime cost rate $\left(c_{d o}^{s-}\right)$.

In the above list, the labor cost and downtime cost strongly depend on the maintenance duration. The longer maintenance duration the higher costs are. In addition, all the costs depend on the nature of the maintenance action (preventive or corrective) and the characteristics of maintained component $i$. Thanks to the above discussions, the cost of a preventive and corrective maintenance actions of component $i$, denoted by $C^{i p}$ and $C^{i c}$ respectively, can be represented under the same following mathematical expression

$$
C^{i-}=\underbrace{\left[C_{s e}^{i-}+C_{s p}^{i-}+\left(1-\pi_{i}\right) C_{s h}^{i-}+\pi_{i} \cdot C_{s h}^{s-}\right]}_{C_{i n d e}^{i-}}+\underbrace{\left[c_{l a}^{i-}+\left(1-\pi_{i}\right) c_{d o}^{i-}+\pi_{i} \cdot c_{d o}^{s-}\right] \cdot \omega^{i-}}_{C_{d e}^{i-}}
$$

where, (a) $\pi_{i}$ is an criticality indicator, $\pi_{i}=1$ if component $i$ is a critical one, otherwise $\pi_{i}=0$ if the component is a redundant one; $(b)$ the total maintenance cost of component $i$ can be divided into two parts: $C_{i n d e}^{i-}$ represents the part which does not depend on the maintenance duration, and $C_{d e}^{i-}$ is the part which depends on the maintenance duration.

When compared to other maintenance cost structures in the literature [31, 30, 24, 26], the proposed model seems to be more general and advantageous:

- It is applied for both preventive and corrective maintenance action.

- Flexible and suitable for a wide range of systems in real applications including complex production systems. Maintenance cost is modeled as a function of maintenance duration $\left(\omega^{i *}\right)$, component's criticality $\left(\pi_{i}\right)$, nature of maintenance action (preventive or corrective), and component's characteristics $(i)$.

- Several specific cost models in the literature can be derived as special cases of the proposed model. For example, in the case of series structure systems $\left(\pi_{i}=1, \forall i\right)$, and negligible maintenance duration $\left(\omega^{i-}=0\right)$, the maintenance cost can be simply calculated by as

$$
C^{i-}=C_{i n}^{i-}=C_{s e}^{i-}+C_{s p}^{i-}+C_{s h}^{s-}
$$

- Different levels of downtime costs, shutdown costs, and setup cost allow to model the economic dependence among components in complex situations, and improve the grouping performance (see more details in the next subsection).

\subsection{Economic dependence and grouping maintenance}

Economic dependence From a practical of view, the total maintenance cost when performing maintenance of several components together may differ from the one when executing maintenance of these components separately. Let $C^{G^{k}}$ be the total maintenance cost of group $G^{k}, C^{G^{k}} \neq \sum_{i \in G^{k}} C^{i-}$. The economic dependence is positive if $C^{G^{k}}<\sum_{i \in G^{k}} C^{i-}$, and otherwise it is negative if $C^{G^{k}}>\sum_{i \in G^{k}} C^{i-}$.

According to our maintenance cost model, the economic dependence may come from: (a) sharing of setup cost leads to positive economic dependence, e.g. the preparation activities including logistic support have to be done once when components are jointly maintained; (b) changing of total shutdown costs can lead to positive 
or negative economic dependence depending on the system structure, e.g. the shutdown cost can be saved when some critical components are jointly maintained; and the cost can be penalized when the maintenance of some non-critical components leads to a complete shutdown of the system; (c) changing of total downtime costs can lead to positive or negative economic dependence depending on the system structure and maintenance duration, e.g. the maintenance duration and therefore the downtime cost can be reduced when the number of repairmen is sufficient to repair multiple components at the same duration. Note that this changing only occurs when the maintenance duration is considered (see subsection 3.3 for further explanations).

Dynamic grouping maintenance approach Dynamic grouping maintenance approach has been developed in $[24,30,13]$ to reduce the maintenance cost by taking advantages of the economic dependence. The proposed approach finds the best way to group PM actions through two main following phases:(a) maintenance optimization at component level. Preventive maintenance activities are individually planned for components by neglecting all their dependencies. The optimal PM frequency of each component is determined by minimizing its long-term maintenance cost rate; (b) grouping optimization at system level. Optimization techniques such as Dynamic Programming and Genetic Algorithm (GA) are used to find the best way to group the tentative individual PM activities in a short-term planning horizon. The best grouping solution has to maximize the profit economic of grouping in the considered short-term planning horizon.

The two above phases are repeated in order to find the optimal grouping plan in the next planning horizons. Given that the planning is done for every finite horizon, the maintenance plan can be dynamically updated whenever dynamic contexts occur [30]. In addition, only PM activities are grouped at specific dates, the logistic support for the maintenance then can be prepared proactively at a lower cost.

Dynamic grouping and impacts of maintenance duration Since almost the dynamic grouping maintenance are planned within finite horizons, the consideration of maintenance (preventive and corrective) duration is important to guarantee the accuracy/optimality of grouping solution. However, it leads to a number of difficulties in grouping modeling and grouping optimization as well. Indeed, at component level, the consideration of maintenance duration may make the finding of the optimal maintenance cycle become more complex and the tentative PM dates could not be fixed over time due to random failures of components. Note that the grouping of changing PM dates is almost impossible. The situation is even more complex at system level where the downtime of one component can influence the functioning of the other ones according to the system structure. The determination of components' age, components' criticality, as well as grouping economic profit are not evident.

To face the mentioned issues, the objectives of the paper are then (i) to propose a grouping maintenance strategy based on analytical methods for multi-component system with redundancy and non-negligible maintenance duration; (ii) to analyze impacts of maintenance duration on maintenance modeling, maintenance optimization (optimality and robustness of grouping maintenance). 


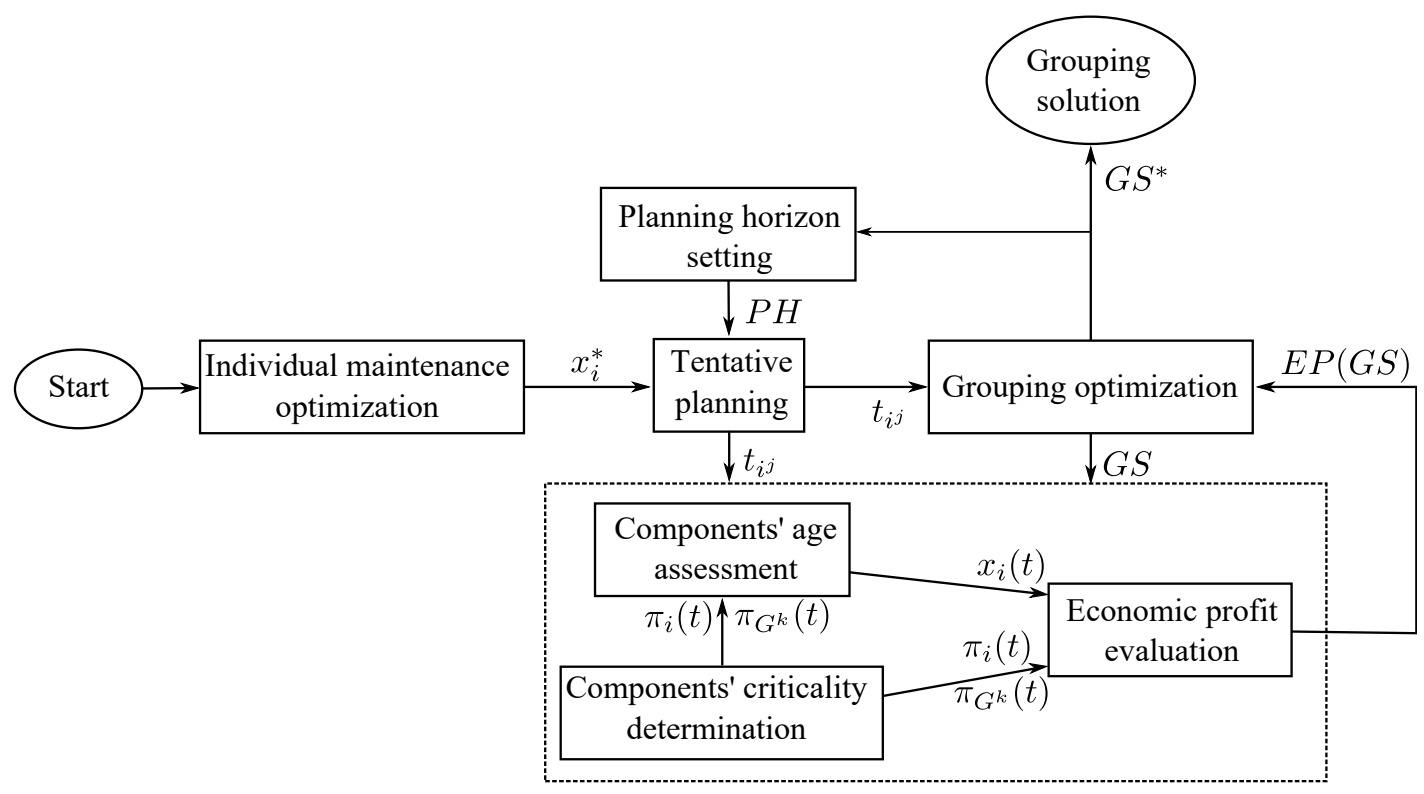

Figure 2: Grouping maintenance approach for multi-component systems with consideration of maintenance duration

\section{Grouping maintenance with considerations of maintenance dura- tion}

In this section, a dynamic grouping maintenance strategy is developed for multi-component systems with taking into account both the maintenance duration and the complexity of system structure. Figure 2 summarizes all main steps of the proposed strategy. Besides basic steps such as individual maintenance or grouping maintenance optimization which are similar to the ones of dynamic grouping strategies proposed in previous works, two additional steps (components' age assessment and components' criticality determination) are developed to overcome the difficulties in grouping modeling and grouping optimization when maintenance duration and complex structure are considered. The two steps are done by proposed analytical methods instead of simulation ones which are usually used in other works, especially when the corrective maintenance duration is non-negligible. Since the grouping process in Figure 2 is proposed based on the analytical methods, it can be applied for online grouping decision-making of multi-component systems with complex structure and non-negligible maintenance duration (both corrective and preventive). For more details, all steps of the proposed grouping maintenance process will be explained and discussed in the next subsections.

\subsection{Individual maintenance optimization}

Components are separately studied to find their optimal preventive maintenance cycle. To do this, component $i$ is considered to be preventively maintained whenever its age reaches a threshold denoted by $x_{i}^{0}$. When it fails between two consecutive PM dates, corrective maintenance is carried to restore it to the "as bad as old" state. The age threshold can be determined by minimizing the long-term maintenance cost rate of the component, denoted $C R_{i}$. Note that the other criteria such as component's availability or component's reliability are certainly can be also used. 


$$
C R_{i}(t)=\lim _{t \rightarrow \infty} \frac{C_{i}(t)}{t}
$$

where $C_{i}(t)$ is the cumulative maintenance cost until time $t$.

By applying renewal theory, $C R_{i}$ can be rewritten as

$$
C R_{i}(s)=\frac{\mathbb{E}\left[C_{i}\left(s_{i}\right)\right]}{\mathbb{E}\left[s_{i}\right]}=\frac{C^{i p}+C^{i c} \cdot \nu_{i}\left(0, s_{i}\right)}{x_{i}^{0}+\omega^{i p}+\omega^{i c} \cdot \nu_{i}\left(0, s_{i}\right)}
$$

where, $\mathbb{E}[*]$ is expected value operator; $s_{i}$ is the renewal cycle of component $i ; \nu_{i}\left(0, s_{i}\right)$ denotes the expected number of failures of component $i$ within the renewal cycle.

Under minimal repair and Weibull failure assumptions, $\nu_{i}\left(0, s_{i}\right)$ can be written as

$$
\nu_{i}\left(0, s_{i}\right)=\int_{0}^{x_{i}} z_{i}(t) d t=\left(\frac{x_{i}^{0}}{\lambda_{i}}\right)^{\beta_{i}}
$$

By substituting Equations (2), (6) into Equation (5), the long-term maintenance cost rate is calculated as

$$
C R_{i}\left(x_{i}^{0}\right)=\frac{C^{i p} \cdot \lambda_{i}^{\beta_{i}}+C^{i c} \cdot\left(x_{i}^{0}\right)^{\beta_{i}}}{\left(x_{i}^{0}+\omega^{i p}\right) \cdot \lambda_{i}^{\beta_{i}}+\omega^{i c} \cdot\left(x_{i}^{0}\right)^{\beta_{i}}}
$$

The optimal value of $x_{i}^{0}$, denoted $x_{i}^{*}$, which minimizes the above long-term maintenance cost rate, can be determined by solving the following equation

$$
\left.\frac{d C R_{i}}{d x_{i}}\right|_{x_{i}^{0}=x_{i}^{*}}=0 \Leftrightarrow C^{i c} \cdot\left(\beta_{i}-1\right) \cdot\left(x_{i}^{*}\right)^{\beta_{i}}+\left(C^{i c} \cdot \omega^{i p}-C^{i p} \cdot \omega^{i c}\right) \cdot \beta_{i} \cdot\left(x_{i}^{*}\right)^{\beta_{i}-1}-C^{i p} \cdot \lambda_{i}^{\beta_{i}}=0
$$

The two following cases are considered:

- Case 1: Both preventive and corrective maintenance duration are neglected [24, 30]. By substituting $\omega^{i p}=\omega^{i c}=0$ in the above equation, we have

$$
C_{i n}^{i c} \cdot\left(\beta_{i}-1\right) \cdot\left(x_{i}^{\overline{P C}}\right)^{\beta_{i}}-C_{i n}^{i p} \cdot \lambda_{i}^{\beta_{i}}=0 \Rightarrow x_{i}^{\overline{P C}}=\lambda_{i} \cdot \sqrt[\beta_{i}]{\frac{C_{i n}^{i p}}{C_{i n}^{i c} \cdot\left(\beta_{i}-1\right)}}
$$

where $x_{i}^{\overline{P C}}$ denotes the optimal PM cycle when preventive and corrective time are neglected.

- Case 2: Only preventive maintenance duration is considered $[13,31,32]$. With $\omega^{i p} \neq 0$, the closedform of $x_{i}^{P \bar{C}}$ (the optimal replacement cycle when preventive maintenance duration is considered) cannot be deduced. Numerical methods are needed to search its approximated value. In Appendix A.1, we demonstrate that $x_{i}^{P \bar{C}} \in\left(0, a_{1}\right]$ with

$$
a_{1}=\lambda_{i} \cdot \sqrt[\beta_{i}]{\frac{C^{i p}}{C_{i n}^{i c} \cdot\left(\beta_{i}-1\right)}}
$$

- Case 3: Both preventive and corrective maintenance duration are considered. Similarly to the previous case, the closed-form of $x_{i}^{P C}$ (the optimal replacement cycle when preventive and corrective time are considered) cannot be obtained. The following propositions are demonstrated in Appendix A.2.

Proposition 1 If $\frac{C^{i c}}{C^{i p}}>\frac{\omega^{i c}}{\omega^{i p}}$, then $x_{i}^{P C}$ exists and is unique in the open interval $\left(0, \min \left(a_{2}, b\right)\right)$ with

$$
a_{2}=\lambda_{i} \cdot \sqrt[\beta_{i}]{\frac{C^{i p}}{C^{i c} \cdot\left(\beta_{i}-1\right)}} ; \quad b=\lambda_{i} \cdot \sqrt[\beta_{i}-1]{\frac{C^{i p}}{\beta_{i} \cdot\left(C^{i c} \cdot \omega^{i p}-C^{i p} \cdot \omega^{i c}\right)}} ;
$$

Proposition 2 If $\frac{C^{i c}}{C^{i p}} \leq \frac{\omega^{i c}}{\omega^{i p}}$, then $x_{i}^{P C}$ exists and is unique in the half-closed interval $\left[\max \left(a_{2}, c\right),+\infty\right)$ with

$$
c=\frac{C^{i p} \cdot \omega^{i c}-C^{i c} \cdot \omega^{i p}}{C^{i c}}
$$




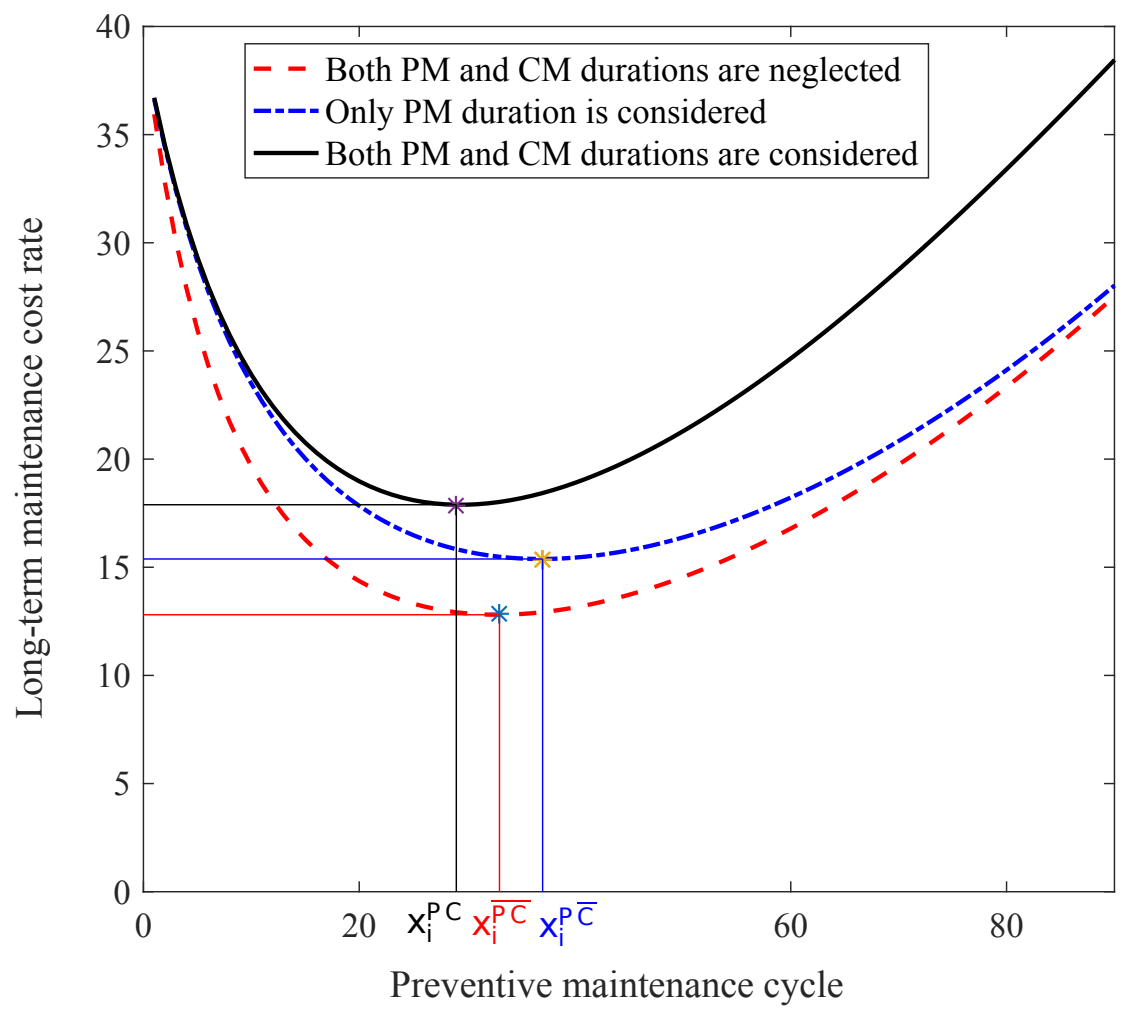

Figure 3: Optimal PM cycles vs Different maintenance duration assumptions

Consider an example of a component $i$ with the following data: $\lambda_{i}=50, \beta_{i}=3, \omega^{i p}=5, \omega^{i c}=3, C^{i p}=547$, $C_{i n}^{i p}=357, C^{i c}=460, C_{i n}^{i c}=205$. Figure 3 represents the evolution of the long-term maintenance cost rate as a function of PM cycle for these above cases. The optimal PM cycles $x_{i}^{\overline{P C}}, x_{i}^{P \bar{C}}, x_{i}^{P C}$ obtained in the three different cases 1, 2, et 3, are not the same. Indeed, when comparing the two Cases 1 and 2, the PM (the replacement) cost in Case 2 is bigger than that in Case 1, while their CM costs are the same. It is then reasonable when $x_{i}^{P \bar{C}}$ is bigger than $x_{i}^{\overline{P C}}$. Similarly, when comparing the two Cases 2 and 3 , the CM cost in Case 3 is bigger than that in Case 2, while the PM costs of the two cases are the same. It is then reasonable when $x_{i}^{P C}$ is smaller than $x_{i}^{P \bar{C}}$. It should be noted that $x_{i}^{\overline{P C}}$ may be bigger or smaller than $x_{i}^{P C}$ depending on the ratio between PM and $\mathrm{CM}$ costs. Figure 3 shows the case where $x_{i}^{\overline{P C}}>x_{i}^{P C}$.

From the above analysis, we can conclude that taking into maintenance duration makes maintenance modeling and optimization more complex. It could be however necessary to guarantee a proper replacement cycle and lower maintenance cost.

Further numerical investigation on the impacts of maintenance duration on individual maintenance optimization will be presented in Section 5.2. Note that the impacts of system structure are not mentioned since the interactions among components are neglected at this step.

\subsection{Tentative maintenance planning.}

Based on the components' nominal PM cycle (age threshold), tentative execution dates of components are determined in a finite planning interval $P H=\left[t_{b}, t_{e}\right] . t_{b}$ is set by the current date and $t_{e}$ is chosen in the way that each component is preventively carried out at least one time within the planning horizon to ensure that all components are taken into account in the maintenance decision-making, see [24, 31]. 
Let $t_{i^{j}}(j \geq 1)$ denote the tentative execution date of the $j^{t h} \mathrm{PM}$ of component $i$, we have

$$
\begin{aligned}
t_{i^{1}} & =x_{i}^{*}-x_{i}\left(t_{b}\right)+y_{i}^{1}+t_{b} \\
t_{i^{j}} & =t_{i^{j-1}}+\omega^{i p}+y_{i}^{j} \text { with } j>1 \text { and } t_{i^{j}} \leq t_{e} \\
t_{e} & =t_{l^{1}} \text { with } t_{l^{1}}=\max _{i} t_{i^{1}} .
\end{aligned}
$$

where, $x_{i}\left(t_{b}\right)$ is the age of component $i$ at the beginning of the planning horizon $t_{b} ; y_{i}^{1}$ and $y_{i}^{j}$ are the cumulative downtimes of component $i$ within intervals $\left(t_{b}, t_{i^{1}}\right)$ and $\left(t_{j^{j-1}}, t_{i^{j}}\right)$ respectively. It is important to note that the cumulative downtimes $y_{i}^{1}$ and $y_{i}^{j}$ depend on both the maintenance duration and the system structure. Regarding to the maintenance duration modelling, the following specific cases are considered.

Case 1: Both CM and PM duration are neglected [24, 30]. The cumulative downtime equals zero. Tentative maintenance dates can be easily determined by using Equation (13) with $y_{i}^{1}=y_{i}^{j}=0$. Note that the system structure has no impact in this case.

Case 2: Only PM duration is considered [13, 31, 32]. The cumulative downtime of component $i$ is different to zero and may depend on the preventive maintenance duration of the other components in the system. As an example, a PM maintenance of component 1 or 3 in the system presented by Figure 1 will lead to shutdowns of component 4. Therefore, the PM duration of components 1 and 3 has to be added to the cumulative downtime of component 4. It is obvious that the dependence is given by the system structure. For series structure systems, this kind of dependence always exists since the maintenance of a component directly leads to a shutdown of the others. The problem becomes more complex in the case of systems with redundancy, the functional dependence among components can be identified by "System's structure analysis" step (see Section 4.1 for more details).

Case 3: Both CM and PM duration are considered. The cumulative downtime of component $i$ may depend on both preventive and corrective maintenance duration of the other components in the system. Since components' failure are random, the cumulative downtime and tentative maintenance dates are likewise not constant. The changing of tentative maintenance dates over time causes difficulties in maintenance optimization and implementation, especially for grouping maintenance. To overcome this issue, an efficient solution is herein proposed. In more details, to establish a tentative maintenance plan with fixed PM dates, a calendar timebased threshold $T_{i}$ is designed and used instead of the age one. In that way, component $i$ is then preventively replaced at every $T_{i}$ units of time without regarding the failures between two consecutive PM activities. $T_{i}$ can be calculated as

$$
T_{i}=\omega^{i p}+x_{i}^{P C}+\omega^{i c} \cdot\left(\int_{0}^{x_{i}^{C M}} z_{i}(t) d t\right)=\omega^{i p}+x_{i}^{P C}+\omega^{i c} \cdot\left(\frac{x_{i}^{P C}}{\lambda_{i}}\right)^{\beta_{i}}
$$

The tentative PM dates can be obtained based on $T_{i}$ as follow

$$
t_{i^{j}}=t_{i^{j-1}}+T_{i} \text { with } j \geq 1 \text { and } t_{i^{j}} \leq t_{e}
$$

where $t_{i^{0}}$ is the execution date of the last PM action before $t_{b}$.

The tentative maintenance planning based on $T_{i}$ has some advantages: (i) fixed PM dates at the same maintenance cost level when compared to the maintenance planning based on $x_{i}^{C M}$; (ii) easier for maintenance 
implementation and management; (iii) can be used for grouping maintenance in the next phase. However, it should be noticed that maintenance planning based on $T_{i}$ is not always better than that based on $x_{i}^{C M}$, especially when components are totally replaced instead of minimally repaired at failures (non-repairable systems). For this case, another solution could be developed in the future research.

\subsection{Economic profit formulation}

Given the tentative maintenance dates of components obtained in the previous phase, this subsection focuses on a formulation of economic profit when several PM actions are jointly performed thanks to the economic dependence among components. For more details, consider a group $G^{k}$ consisting of $m$ PM activities which are executed together. The economic profit of $G^{k}$, denoted $E P\left(G^{k}\right)$, contains five following parts.

Saving of the preventive setup cost, denoted by $\mathbf{S}_{\mathbf{G}^{\mathbf{k}}}$. The saving comes from the sharing of setup operations for executing $m$ PM activities together. For example, scaffolding erecting and machine opening may have to be done only once for the maintenance of several components.

$$
S_{G^{k}}=\sum_{i^{j} \in G^{k}} C_{s e}^{i p}-C_{s e}^{G^{k}}
$$

where $C_{s e}^{G^{k}}$ is the setup cost of group $G^{k}$. According to different levels of economic dependence among components in the group, this group setup cost can be varied.

$$
\min _{i^{j} \in G^{k}} C_{s e}^{i p} \leq C_{s e}^{G^{k}} \leq \sum_{i^{j} \in G^{k}} C_{s e}^{i p}
$$

Penalty cost due to the change of nominal PM dates, denoted by $\Delta \mathbf{H}_{\mathbf{G}^{\mathrm{k}}}^{\mathbf{1}}$. As a consequence of maintenance grouping, the PM execution date of several components may be either advanced or delayed regarding to their tentative PM date. These movements of execution dates result in changing the expected number of failures in short-term, and shifting all future maintenance dates in long-term (see [24] for more explanations). The penalty cost is then calculated as

$$
\Delta H_{G^{k}}^{1}=\sum_{i^{j} \in G^{k}}\left\{C^{i c} \cdot\left[\nu_{i}\left(0, t_{i^{j}}\right)-\nu_{i}\left(0, t_{G^{k}}\right)\right]-C R_{i}\left(x_{i}^{P C}\right) \cdot\left(t_{i^{j}}-t_{G^{k}}\right)\right\}
$$

$\Delta H_{G^{k}}^{1}$ depends on both CM and PM duration.

Cost associated with the change of system shutdown times, denoted by $\Delta \mathbf{H}_{\mathbf{G}^{\mathrm{k}}}^{\mathbf{2}}$. This cost indicates that the number of times that the system is shut down due to the PM of some components is not equal to that of their group.

$$
\Delta H_{G^{k}}^{2}=\left[\sum_{i^{j} \in G^{k}} \pi_{i}-\pi_{G^{k}}\right] \cdot C_{s h}^{s p}
$$

$\Delta H_{G^{k}}^{2}$ depends on the system structure. Its maximum and minimum values are reached in case of series structures $\left(\pi_{i}=1, \forall i=1, \ldots n\right)$, and parallel structures $\left(\pi_{i}=0, \forall i=1, \ldots n\right)$ respectively.

$$
-C_{s h}^{s p} \leq \Delta H_{G^{k}}^{2} \leq(m-1) \cdot C_{s h}^{s p}
$$

Note however that $\Delta H_{G^{k}}^{2}$ does not depend on neither PM duration nor CM duration. 


\section{Cost related to the change system downtime duration, denoted by $\Delta \mathrm{H}_{\mathrm{G}^{\mathrm{k}}}^{3}$.}

$$
\Delta H_{G^{k}}^{3}=\left[\sum_{i^{j} \in G^{k}} \pi_{i} \cdot \omega^{i p}-\pi_{G^{k}} \cdot \omega_{G^{k}}\right] \cdot c_{d o}^{s p}
$$

This part is similar to $\Delta H_{G^{k}}^{2}$, except that it depends on the PM duration of individual components $\omega^{i p}$, and that of their group $\omega_{G^{k}}$. It should be noticed that the group's PM duration may depend on the number of repairman [32].

$$
\max _{i^{j} \in G^{k}} \omega^{i p} \leq \omega_{G^{k}} \leq \sum_{i^{j} \in G^{k}} \omega^{i p}
$$

Costs related to the increase of system failure frequency during PM duration, denoted by $\Delta \mathbf{H}_{\mathbf{G}^{\mathbf{k}}}^{\mathbf{4}}$. During the PM time of a redundant component or non-critical group, the system is still operating. However, its redundancy level is decreased, and in the worst-case scenario, one or several redundant components can play a critical role for the system functioning. Consequently, failures of these components result in extra system's shutdown and downtime costs.

$$
\begin{aligned}
\Delta H_{G^{k}}^{4}= & \sum_{i^{j} \in G^{k}}\left(1-\pi_{i}\right) \cdot \sum_{l \in L_{i}}\left(C_{s h}^{s c}+c_{d o}^{s c} \cdot \omega^{l c}\right) \cdot \nu_{p}\left(t_{i^{j}}, t_{i^{j}}+\omega^{i p}\right) \\
& -\left(1-\pi_{G^{k}}\right) \cdot \sum_{q \in Q_{G^{k}}}\left(C_{s h}^{s c}+c_{d o}^{s c} \cdot \omega^{q c}\right) \cdot \nu_{q}\left(t_{G^{k}}, t_{G^{k}}+\omega_{G^{k}}\right)
\end{aligned}
$$

where $Q_{G^{k}}$ and $L_{i}$ denote the sets of redundant components playing a critical role during the PM of group $G^{k}$ and component $i$ respectively. For example, consider the system structure in Figure 1, when two components 2 and 5 are separately maintained, we have $L_{2}=L_{3}=\emptyset$. Otherwise, when they are jointly maintained $G^{k}=\{2,5\}$, components 3 and 4 become critical ones, $Q_{G^{k}}=\{3,4\}$.

Grouping economic profit of group $\mathbf{G}^{\mathbf{k}}$. From the above analysis, the economic profit of group $G^{k}$ is calculated by

$$
E P\left(G^{k}\right)=S_{G^{k}}+\Delta H_{G^{k}}^{1}+\Delta H_{G^{k}}^{2}+\Delta H_{G^{k}}^{3}+\Delta H_{G^{k}}^{4}
$$

It should be noted that most parts of $E P\left(G^{k}\right)$, except $S_{G^{k}}$, are determined based on the knowledge about the component's conditions (its criticality and its age) at different instants in both tentative and grouped maintenance plans. According to different assumptions about the maintenance duration, the determination of $E P\left(G^{k}\right)$ and component's conditions could be different. For more details, the following cases are considered

Case 1: Both CM and PM time are neglected [24, 30]. It is clear that $\Delta H_{G^{k}}^{3}=\Delta H_{G^{k}}^{4}=0$ and consequently $\operatorname{EP}\left(G^{k}\right)=S_{G^{k}}+\Delta H_{G^{k}}^{1}+\Delta H_{G^{k}}^{2}$. The criticality of components is constant and easily determined. The age of components is equal to the calendar time. The calculation of $\operatorname{EP}\left(G^{k}\right)$ is even more simple for series structure since all components and groups of components in a series structure are always critical $\pi_{i}=\pi_{G^{k}}=1$, and therefore $\Delta H_{G^{k}}^{2}$ can be directly calculated as $\Delta H_{G^{k}}^{2}=(m-1) \cdot C_{s h}^{s p}$, where $m$ is the number of components in group $G^{k}$.

Case 2: Only PM time is considered $[13,31,32]$. When the PM time is not equal to zero, $\Delta H_{G^{k}}^{3}$ and $\Delta H_{G^{k}}^{4}$ exist. The calculation of $E P\left(G^{k}\right)$ is slightly complex since the criticality of one component can be changed within the PM time of other ones, and the component's age does not equal the calendar time. 
Fortunately, the PM dates are known in advance, the PM time is then can be proactively integrated in the calculations of economic profit. Regarding the system structure, the calculation is quite simple in the case of series structure where components and groups are always critical even when PM time is considered. Otherwise, for redundant structures, "components' criticality determination" method is needed to determined the criticality of components during PM periods (see Subsection 4.1 for more details).

Case 3: Both CM and PM time are considered. In this situation, the analytic calculation of $E P\left(G^{k}\right)$ becomes more complex. Since component's failures are random, the determination of component's age in presence of CM duration is not evident. In addition to the randomness of the cumulative CM duration, the operational dependence among components in a system structure makes the calculation of their age cannot be done separately. To deal with the problem, most of papers in the literature have been proposed calculation methods based on Monte Carlo Simulation. In the paper, an analytical method named "components' age assessment" is developed and presented in Subsection 4.2.

\subsection{Grouping optimization}

This section is devoted to firstly define a grouping solution and grouping optimization problems. The solutions for grouping optimization problems are then discussed in different cases. Before the grouping optimization can be mathematically defined, two following notations should be firstly clarified.

A grouping solution (grouping structure), denoted by $G S$, is a partition of $N$ preventive maintenance activities in $P H$. The partition of $\{1, \ldots, N\}$ is a collection of $K$ mutually exclusive groups $G S=\left\{G^{1}, \ldots, G^{K}\right\}$ which cover all $N$ preventive maintenance activities.

$$
G^{l} \cap G^{k}=\emptyset, \forall l \neq k \text { and } G^{1} \cup G^{2} \cup \ldots \cup G^{K}=\{1, \ldots, N\}
$$

Since one component can be preventively maintained several times within a considered planning horizon , the number of PM activities $N$ then can be bigger than that of components $n$.

Economic profit of a grouping solution is equal to the sum of economic profits of all groups in a grouping struture.

$$
E P T=\sum_{k=1}^{K} E P\left(G^{k}, t_{G^{k}}\right)=\sum_{k=1}^{K} S_{G^{k}}+\Delta H_{G^{k}}^{1}+\Delta H_{G^{k}}^{2}+\Delta H_{G^{k}}^{3}+\Delta H_{G^{k}}^{4}
$$

Grouping optimization problem is then defined as the finding of the optimal grouping structure $G S^{*}=$ $\left\{G^{1 *}, \ldots, G^{K *}\right\}$ and the optimal execution dates of each group in the optimal grouping structure $T_{G S}^{*}=\left\{t_{G^{1 *}}^{*}, \ldots, t_{G^{K *}}^{*}\right\}$ who maximize the total economic profit in the short-term planning horizon.

$$
\left[G S^{*}, T_{G S}^{*}\right]=\underset{G S, T_{G S}}{\arg \max } \operatorname{EPT}\left(G S, T_{G S}\right)=\underset{G^{k}, t_{G^{k}}}{\arg \max } \sum_{k=1}^{K} E P\left(G^{k}, t_{G^{k}}\right)
$$

The grouping optimization problem can be divided into two sub-problems: grouping structure optimization and groups' execution date optimization.

\subsubsection{Grouping structure optimization.}

The optimal grouping structure can be found in maximizing the total grouping economic profit.

$$
G S^{*}=\underset{G S}{\arg \max } \operatorname{EPT}(G S)
$$


Given $N$ maintenance activities in a considered horizon $P H$, there are $2^{N}-1$ possible groups [9]. The finding of optimal grouping structure is a NP-complete problem. According to different considered hypothesis, appropriate optimization techniques must can be selected. Without considering the system structure and CM duration, dynamic programming becomes an appropriate choice, see [9, 31]. Needed, the use of dynamic programming is based on theorem of consecutive maintenance activities which implies that, in an optimal grouping solution, maintenance activities are executed in the order of their tentative maintenance dates. As a consequence, the maximum number of groups to be considered reduces from $2^{N}-1$ to $\frac{1}{2} N(N+1)$. However, when considering the $\mathrm{CM}$ and PM duration and/or the system structure, $\Delta H_{G^{k}}^{2}, \Delta H_{G^{k}}^{3}, \Delta H_{G^{k}}^{4}$ are usually not equal to zero. theorem of consecutive maintenance activities does not hold. The maximum number of groups can not be reduced. Metaheuristic optimization techniques such as Genetic Algorithm (GA), Particle Swarm Optimization (PSO) need to be used to find the best grouping solution in a reasonable calculation time. An implementation of the GA for the grouping maintenance optimization can be found in $[30,13]$. Note that the optimality of the grouping solution provided by the metaheuristic optimization techniques may not always be guaranteed.

\subsubsection{Groups' execution date optimization.}

For each grouping solution, the execution dates of its groups have to be optimized. Given that $S_{G^{k}}, \Delta H_{G^{k}}^{2}$ and $\Delta H_{G^{k}}^{3}$ do not depend on $t_{G^{k}}$, the optimal execution date is determined as

$$
t_{G^{k}}^{*}=\underset{t_{G^{k}}}{\arg \max } \operatorname{EP}\left(G^{k}, t_{G^{k}}\right)=\underset{t_{G^{k}}}{\arg \min }\left(\Delta H_{G^{k}}^{1}+\Delta H_{G^{k}}^{4}\right)
$$

Two specific following situations are considered.

Both CM and PM duration are neglected $[24,30] . \Delta H_{G^{k}}^{4}$ is then equal to zero. $t_{G^{k}}^{*}$ can be easily determined by minimizing $\Delta H_{G^{k}}^{1}$.

$$
t_{G^{k}}^{*}=\underset{t_{G^{k}}}{\arg \min } \Delta H_{G^{k}}^{1}
$$

CM and/or PM duration are considered. In this case, $\Delta H_{G^{k}}^{4}$ is not equal to zero. In addition, with the presence of maintenance duration, the tentative maintenance dates of one component depends on that of other components in the system. The execution dates of groups in a grouping solution are therefore interdependent and can not be determined separately. The problem should be redefined as

$$
\left\{t_{G^{1}}^{*}, t_{G^{2}}^{*}, \ldots, t_{G^{K}}^{*}\right\}=\underset{\left\{t_{G^{1}}, t_{G^{2}}, \ldots, t_{G} K\right.}{\arg \min } \sum_{k=1}^{K}\left(\Delta H_{G^{k}}^{1}+\Delta H_{G^{k}}^{4}\right)
$$

\section{Analytical methods for evaluating components' criticality and age in presence of maintenance duration}

In the presence of maintenance duration, determinations of components' criticality and components' age are not evident. To solve the problem, two analytical methods are herein proposed and presented. In addition, impacts of maintenance duration in maintenance modelling and optimization are summarized at the end of this section. 


\subsection{Components' criticality determination}

This analytical method is developed to analyze the operational dependence among components of redundant structure systems and determine the criticality of components or group of components within specific periods of time such as PM periods. The determined components' criticality will be used in different steps including tentative planning, components' age assessment, and economic profit evaluation (see in Figure 2). Note that the components' criticality determination step can be skipped in case of series structures where components or groups of components are always critical. Before the method could be developed for redundant structures, the following basic notations are firstly recalled [34]: (a) a path set is a set of components which by functioning ensures that the system is functioning. A path set is said to be minimal if it cannot be reduced without loosing its status as a path set; (b) a cut set is a set of components which by failing causes the system to fail. A cut set is said to be minimal if it cannot reduced without loosing its status as a cut set.

Let $U=\{1, \ldots, n\}, P=\left\{p_{1}, \ldots, p_{n_{p}}\right\}$ and $C=\left\{c_{1}, \ldots, c_{n_{c}}\right\}$ respectively denote the set of components, set of minimal path sets and set of minimal cut sets of the system when all components are functioning. The operational dependence among components within a specific interval $I_{v}$ can be identified as follows:

- Determine the set of components which are preventively maintained in $I_{v}$, denoted $G_{v}$, according to the considered maintenance plan (individual or grouped).

- Identify the set of all functioning components in $I_{v}$, denoted $O_{v}$, by firstly updating $P$. The updating is done by removing all minimal path sets which contain at least one component in $G_{v}$. $O_{v}$ is then the union of all minimal path sets in $P^{\prime}$ which is the update of $P$.

- Identify the set of all components which are not functioning, denoted $N O_{v}$. NO $v$ contains components which are not in $O_{v}: N O_{v}=U \backslash O_{v}$.

- Identify the set of all components which are in the idle state: $D_{v}=N O_{v} \backslash G_{v}$.

- Identify the set of all components which are critical for the system functioning in $I_{v}$ denoted by $A_{v}$. To this purpose, each minimal cut set of $C$ is firstly updated by eliminating all common elements between it and $N O_{v}$. Let $C^{\prime}$ be the update of $C$. All first order minimal cut sets in $C^{\prime}$, which contain only one component, then make up $A_{v}$.

- Identify the impact of other components on the functioning of a component $f$ in $O_{v}$. The functioning of component $f$ can be interrupted due to the corrective maintenance of one of components in $B_{v}^{f}$ containing: (i) critical component $i$ in $A_{v}$ and $i \neq f$; (ii) redundant component $j$ which is not in $A_{v}$ and $j \neq f$ if $P_{f}^{\prime} \subset P_{j}^{\prime}$, where $P_{j}^{\prime}$ and $P_{f}^{\prime}$ are the sets of minimal path sets which are in $P^{\prime}$ and contain component $j$ and $f$ respectively.

For an easier understanding of this step, operational dependence analysis is done for interval $I_{5}$ in Figure 4 as an example. Given the structure in the figure, we have $U=\{1,2,3,4\}, P=\left\{p_{1}, p_{2}\right\}, p_{1}=\{1,2\}, p_{2}=\{1,3,4\}$, $C=\left\{c_{1}, c_{2}, c_{3}\right\}, c_{1}=\{1\}, c_{2}=\{2,3\}, c_{3}=\{2,4\}$. The operational dependence among components are identified as follows:

- Within $I_{5}$, only component 4 is preventively maintained at $t_{4^{1}}$, therefore $G_{5}=\{4\}$.

- Update $P: P^{\prime}=\left\{p_{1}\right\}, O_{5}=\{1,2\}$.

- Identify non-functioning components: $N O_{5}=\{3,4\}$.

- Identify idle components: $D_{5}=\{3\}$.

- Update minimal cut sets: $C^{\prime}=\left\{c_{1}^{\prime}, c_{2}^{\prime}\right\}, c_{1}^{\prime}=\{1\}, c_{2}^{\prime}=\{2\}$. The set of critical components: $A_{5}=\{1,2\}$. 
- Identify the impact of other components on the functioning of components in $O_{v}: B_{2}^{1}=\{2\}$ and $B_{2}^{2}=\{1\}$.

\subsection{Components' age assessment}

This analytical method is developed to determine the age of components at different instants in tentative and grouped maintenance plans when the CM time is considered (see in Figure 2). Since the consideration of both criticality and age changes at the same time seems to be impossible, the general idea of the proposed method is to find the way that allows to deal with these changes separately. In more detail, the analytical method contains three main following steps:

Step 1: Maintenance plan division for removing impacts of criticality's change. In this step, the maintenance plan (tentative or grouped maintenance plan) is divided into sub-intervals such that the system structure in each sub-interval does not change. A partition of a maintenance plan $P I=\left[t_{b}, t_{e}\right]$ containing $V$ sub-intervals $\left(I_{1}, I_{2}, \ldots, I_{V}\right)$ is the output of this step.

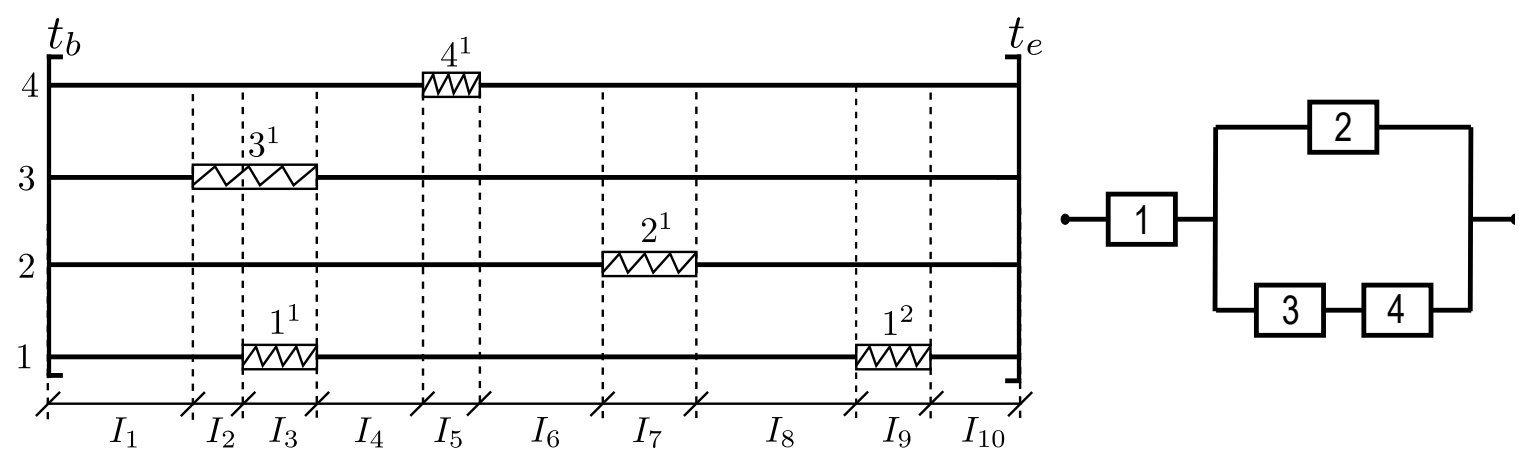

Figure 4: The division of individual maintenance plan

Consider an example of a 4-component system, the individual maintenance plan $P I=\left[t_{b}, t_{e}\right]$ contains 5 PM activities can be divided into 10 sub-intervals $\left(I_{1}, I_{2}, \ldots, I_{10}\right)$ as shown in Figure 4 . The system structure remains unchanged in each sub-interval. Indeed, for example, in sub-interval $I_{5}$, component 4 is preventively maintained, the structure in this sub-interval contains only two components 1 and 2 connected in series. Otherwise, in subinterval $I_{7}$, component 2 is preventively maintained, the system structure in this sub-interval is composed of three components 1, 3, 4 connected in series. It is clearly that in each sub-interval, when the system structure does not change, the criticality of components in this sub-interval is also fix.

Step 2: Operational dependence analysis in sub-intervals. The objective of this step is to determine the operational dependence among functioning components in each above sub-interval $I_{v}$. This step is done by the proposed method presented in Subsection 4.1 .

Step 3: Age updating in sub-intervals. Based on the obtained results from the analysis of operational dependence, proper age-updated actions are carried out for each group of components. Let $x_{i}\left(I_{v}^{b}\right)$ and denote the age of component $i$ at the beginning of the considered sub-interval $I_{v}$. The age of the component at the ending of $I_{v}$, denoted $x_{i}\left(I_{v}^{e}\right)$, is calculated as follow

- $x_{i}\left(I_{v}^{e}\right)=0$ if $i \in G_{v}$. 
- $x_{i}\left(I_{v}^{e}\right)=x_{i}\left(I_{v}^{b}\right)$ if $i \in D_{v}$.

- For components in $O_{v}$, the age is updated by solving the following equation system.

$$
I_{v}^{e}=I_{v}^{b}+\left[x_{i}\left(I_{v}^{e}\right)-x_{i}\left(I_{v}^{b}\right)\right]+\omega^{i c} \cdot \nu_{i}\left(I_{v}^{b}, I_{v}^{e}\right)+\sum_{j \in B_{v}^{i}} \omega^{j c} \cdot \nu_{j}\left(I_{v}^{b}, I_{v}^{e}\right), \forall i \in O_{v}
$$

Reconsider interval $I_{5}$ in Figure 4, we have $G_{5}=\{4\}$, then $x_{4}\left(I_{5}^{e}\right)=0 ; D_{5}=\{3\}$, then $x_{3}\left(I_{5}^{e}\right)=x_{3}\left(I_{5}^{b}\right)$; $O_{5}=\{1,2\}, B_{2}^{1}=\{2\}$ and $B_{2}^{2}=\{1\}$.

$$
I_{5}^{e}=I_{5}^{b}+\left[x_{1}\left(I_{5}^{e}\right)-x_{1}\left(I_{5}^{b}\right)\right]+\omega^{1 c} \cdot \nu_{1}\left(I_{5}^{b}, I_{5}^{e}\right)+\omega^{2 c} \cdot \nu_{2}\left(I_{5}^{b}, I_{5}^{e}\right) .
$$

\subsection{Summary of the impacts of maintenance duration on maintenance modelling and optimization}

In the above sections, dynamic grouping strategy has been developed for multi-component systems with redundancy taking into account both PM and CM duration. In addition, at each step of the developed grouping strategy, the impacts of maintenance duration on maintenance modeling and maintenance optimization are analyzed for the different cases. In this subsection, these mentioned impacts are summarized to give readers their complete picture. The summary is reported in Table 2 . 


\begin{tabular}{|c|c|c|c|c|}
\hline \multicolumn{2}{|c|}{ Maintenance modeling and optimization } & $\begin{array}{l}\text { Both } \mathrm{CM} \text { and PM duration } \\
\quad \text { are neglected (Case 1) }\end{array}$ & $\begin{array}{l}\text { Only PM duration } \\
\text { is considered (Case 2) }\end{array}$ & $\begin{array}{l}\text { Both PM and CM duration } \\
\text { are considered (Case } 3 \text { ) }\end{array}$ \\
\hline \multirow{5}{*}{$\begin{array}{l}\text { Maintenance } \\
\text { modeling }\end{array}$} & Component age & $x_{i}(t)=t$ & \multicolumn{2}{|c|}{$x_{i}(t) \neq t$} \\
\hline & Failure behavior & $z_{i}(t)=\frac{\beta_{i}}{\lambda_{i}}\left(\frac{x_{i}}{\lambda_{i}}\right)^{\beta_{i}-1}$ & \multicolumn{2}{|c|}{$z_{i}(t)=\frac{\beta_{i}}{\lambda_{i}}\left(\frac{x_{i}}{\lambda_{i}}\right)^{\beta_{i}-1}$} \\
\hline & Preventive maintenance cost & $C^{i p}=C_{i n}^{i p}$ & \multicolumn{2}{|c|}{$C^{i p}=C_{i n}^{i p}+c_{d e}^{i p} \cdot \omega^{i p}$} \\
\hline & Corective maintenance cost & $C^{i c}=C_{i n}^{i c}$ & $C^{i c}=C_{i n}^{i c}$ & $C^{i c}=C_{i n}^{i c}+c_{d e}^{i c} \cdot \omega^{i c}$ \\
\hline & Economic dependence & $\begin{array}{l}\text { (1) Sharing of setup cost } \\
\text { (2) Changing of total shutdown costs }\end{array}$ & \multicolumn{2}{|c|}{$\begin{array}{l}\text { (1) Sharing of setup cost } \\
\text { (2) Changing of total shutdown costs } \\
\text { (3) Changing of total downtime costs }\end{array}$} \\
\hline \multirow{2}{*}{$\begin{array}{l}\text { Individual } \\
\text { maintenance } \\
\text { optimization }\end{array}$} & Optimal PM cycle & Closed form: $x_{i}^{\overline{P C}}=\lambda_{i} \cdot{ }^{\beta_{i}} \sqrt{\frac{C^{i p}}{C^{i c} \cdot\left(\beta_{i}-1\right)}}$ & \multicolumn{2}{|c|}{ No closed form } \\
\hline & Tentative maintenance planning & Directly based on age threshold $x_{i}^{\overline{P C}}$ & Based on age threshold $x_{i}^{P \bar{C}}$ & Based on calendar threshold $T_{i}$ \\
\hline \multirow{2}{*}{$\begin{array}{l}\text { Grouping } \\
\text { economic } \\
\text { profit }\end{array}$} & Grouping economic profit formulation & $E P\left(G^{k}\right)=S_{G^{k}}+\Delta H_{G^{k}}^{1}+\Delta H_{G^{k}}^{2}$ & \multicolumn{2}{|c|}{$E P\left(G^{k}\right)=S_{G^{k}}+\Delta H_{G^{k}}^{1}+\Delta H_{G^{k}}^{2}+\Delta H_{G^{k}}^{3}+\Delta H_{G^{k}}^{4}$} \\
\hline & Grouping economic profit calculation & Quite simple & Components' criticality determination is needed & $\begin{array}{l}\text { Both components' criticality determin } \\
\text { and component's age assessment are } n\end{array}$ \\
\hline \multirow{3}{*}{$\begin{array}{l}\text { Grouping } \\
\text { maintenance } \\
\text { optimization }\end{array}$} & \multirow{2}{*}{ Grouping structure optimization } & $\begin{array}{l}\text { (1) Series structures: } \frac{1}{2} N(N+1) \text { possible groups } \\
\text { solved by exact methods (dynamic programming) }\end{array}$ & \multirow{2}{*}{\multicolumn{2}{|c|}{$2^{N}-1$ possible groups; solved by meta-heuristic methods (Genetic algorithm) }} \\
\hline & & $\begin{array}{c}\text { (2) Redundant structures: } 2^{N}-1 \text { possible groups } \\
\text { solved by meta-heuristic methods (Genetic algorithm) }\end{array}$ & & \\
\hline & Group's execution date optimization & $\begin{array}{l}\text { Group's execution dates can be determined separately } \\
\qquad t_{G^{k}}^{*}=\arg \min _{t_{G^{k}}} \Delta H_{G^{k}}^{1}\end{array}$ & \multicolumn{2}{|c|}{$\begin{array}{c}\text { Group's execution dates have to be jointly determined } \\
\left\{t_{G^{1}}^{*}, t_{G^{2}}^{*}, \ldots, t_{G^{K}}^{*}\right\}=\arg \min _{\left\{t_{G^{1}}, t_{G^{2}}, \ldots, t_{G}\right\}} \sum_{k=1}^{K}\left(\Delta H_{G^{k}}^{1}+\Delta H_{G^{k}}^{4}\right)\end{array}$} \\
\hline
\end{tabular}

Table 2: Impacts of maintenance duration on maintenance modelling and optimization 


\section{$5 \quad$ Numerical examples}

The aim of this section is to show how the proposed grouping strategy can be used for the maintenance planning through a numerical study. The performance of the proposed approach is highlighted by different sensitivity analyses. In addition, the impacts of maintenance duration on maintenance plan and cost are also investigated.

\subsection{Given data}

Consider a distillation system containing 6 principle components [35]. The reliability block diagram of this system is shown in Figure 5. In the system, three pumps A, B, and C are configured in parallel to ensure the system functioning. According to the component classification, the criticality of components $\pi_{i}$ is determined and reported in Table 3.

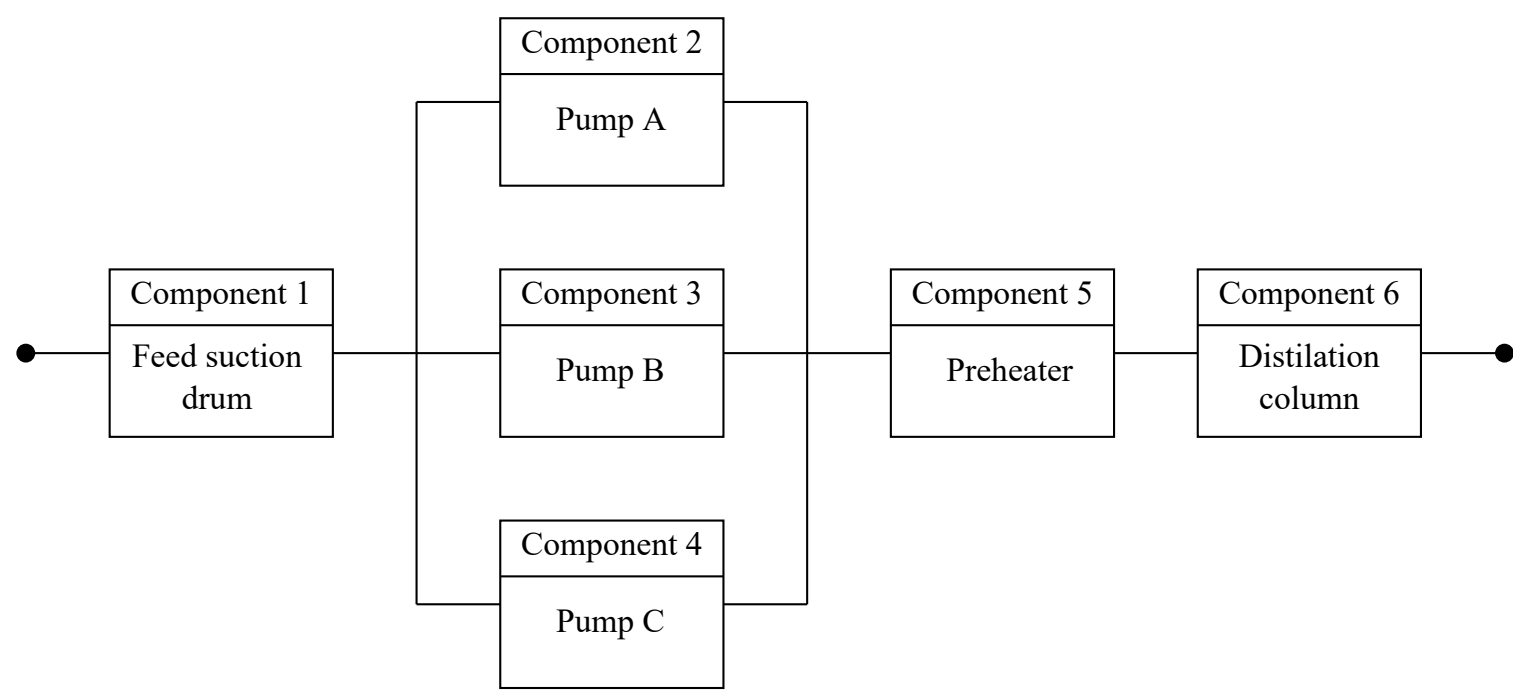

Figure 5: Reliability block diagram of a distillation system containing 6 principle components

Given a number of challenges encountered during the data collection and data processing, we decided to use the generated data. The data of the considered system are generated with respect to the real application constraints. Indeed, it is natural that setup cost, shutdown cost, downtime cost rate, and labor cost rate of CM are bigger than that of PM. They are then generated by the following ways: $C_{s c}^{i c}=C_{s c}^{i p}+3 ; C_{s h}^{i c}=C_{s h}^{i p}+3$; $c_{d o}^{i c}=c_{d o}^{i p}+5 ; c_{l a}^{i c}=c_{l a}^{i p}+2$. At the system level, when the maintenance of a component leads to system non-functioning, additional costs have to be counted for the maintenance of the component: $C_{s h}^{s p}=C_{s h}^{i p}+5$; $c_{d o}^{s p}=c_{d o}^{i p}+30 ; C_{s h}^{s c}=C_{s h}^{i c}+8 ; c_{d o}^{s c}=c_{d o}^{i c}+50$. All the generated data related to the maintenance costs and the failure behavior of components are reported in Table 3 .

In the next subsections, we present in more details how the developed dynamic grouping strategy can be applied to schedule the maintenance of the above system with taking into account both PM and CM duration. In addition, at every step of the proposed grouping strategy, sensitivity analyses are carried out to underline the impacts of maintenance duration and system structure types on the maintenance modeling and maintenance optimization. 
Table 3: Given data at the component level.

\begin{tabular}{clllllllllllr}
\hline Components & $\pi_{i}$ & $\lambda_{i}$ & $\beta_{i}$ & $C_{s c}^{i p}$ & $C_{s p}^{i p}$ & $C_{s p}^{i c}$ & $C_{s h}^{i p}$ & $c_{l a}^{i p}$ & $c_{d o}^{i p}$ & $\omega^{i p}$ & $\omega^{i c}$ & $t_{i}$ \\
\hline 1 & 1 & 353 & 2.05 & 5 & 300 & 15 & 2 & 10 & 05 & 3 & 3.0 & 100 \\
2 & 0 & 180 & 1.85 & 7 & 450 & 20 & 4 & 12 & 08 & 4 & 2.5 & 150 \\
3 & 0 & 217 & 1.87 & 3 & 500 & 22 & 2 & 15 & 02 & 4 & 2.5 & 255 \\
4 & 0 & 217 & 2.00 & 2 & 380 & 18 & 3 & 10 & 12 & 4 & 2.5 & 10 \\
5 & 1 & 250 & 1.65 & 3 & 250 & 45 & 1 & 08 & 10 & 5 & 2.8 & 50 \\
6 & 1 & 384 & 2.00 & 7 & 300 & 33 & 2 & 11 & 06 & 3 & 2.6 & 100 \\
\hline
\end{tabular}

Table 4: Individual maintenance optimization results.

\begin{tabular}{cllllll}
\hline Components & $x_{i}^{\overline{P C}}$ & $x_{i}^{P \bar{C}}$ & $x_{i}^{P C}$ & $C R_{i}\left(x_{i}^{\overline{P C}}\right)$ & $C R_{i}\left(x_{i}^{P \bar{C}}\right)$ & $C R_{i}\left(x_{i}^{P C}\right)$ \\
\hline 1 & 988.4 & 1175.0 & 458.1 & 2.4868 & 2.8123 & 1.8810 \\
2 & 768.4 & 833.1 & 488.6 & 2.5620 & 2.6373 & 2.3677 \\
3 & 1005.5 & 1071.2 & 631.4 & 2.0968 & 2.1467 & 1.9245 \\
4 & 790.7 & 872.4 & 476.2 & 2.1991 & 2.3053 & 1.9539 \\
5 & 764.6 & 1130.0 & 468.0 & 2.8270 & 3.2416 & 2.6351 \\
6 & 909.3 & 1091.6 & 521.5 & 1.9936 & 2.2071 & 1.7252 \\
$\sum_{i=1}^{6} C R_{i}$ & - & - & - & $\mathbf{1 4 . 1 6 5 3}$ & $\mathbf{1 5 . 3 5 0 3}$ & $\mathbf{1 2 . 4 8 7 5}$ \\
\hline
\end{tabular}

\subsection{Individual maintenance optimization}

The objective of the first step is to determine the optimal replacement cycle for each component by neglecting the interactions among components. The optimal replacement cycle is determined for the different cases by using the equations $1,2,3,8,9,10,11,12$. From the obtained results shown in Table 4, we can conclude that the taking into account both PM and CM duration is necessary to guarantee the optimality of replacement cycles and the performance of the individual maintenance optimization. Indeed, the consideration of CM time makes the optimal replacement cycle values may vary between $45 \%$ to $87 \%$ according to different components. In addition, the use of $x_{i}^{P \bar{C}}$ and $x_{i}^{\overline{P C}}$ will lead to higher system maintenance cost rates which are $22.93 \%$ and $13.44 \%$ more expensive than that of $x_{i}^{P C}$.

An other interesting remark is that, given the presence of both PM and CM duration, the taking into account only the PM duration in the maintenance optimization models does not have too much of meaning. In our case, it even causes the higher system maintenance cost rate $\left(C R_{s y s}\left(x_{i}^{P \bar{C}}\right)=15.3503\right)$ when compared to the case where both PM and CM duration are neglected $\left(C R_{s y s}\left(x_{i}^{\overline{P C}}\right)=14.1653\right)$.

To have a more complete view about the impacts of the maintenance duration, especially CM duration on the individual maintenance optimization, a sensitivity analysis was carried out on component 5 . In this analysis, the PM duration $\omega^{5 p}$ was fixed at 5 , and $\mathrm{CM}$ duration $\omega^{5 c}$ is varied from 0.5 to 5 . The optimal replacement cycle and the average maintenance cost rate of the component are calculated and plotted in Figure 6 for different 

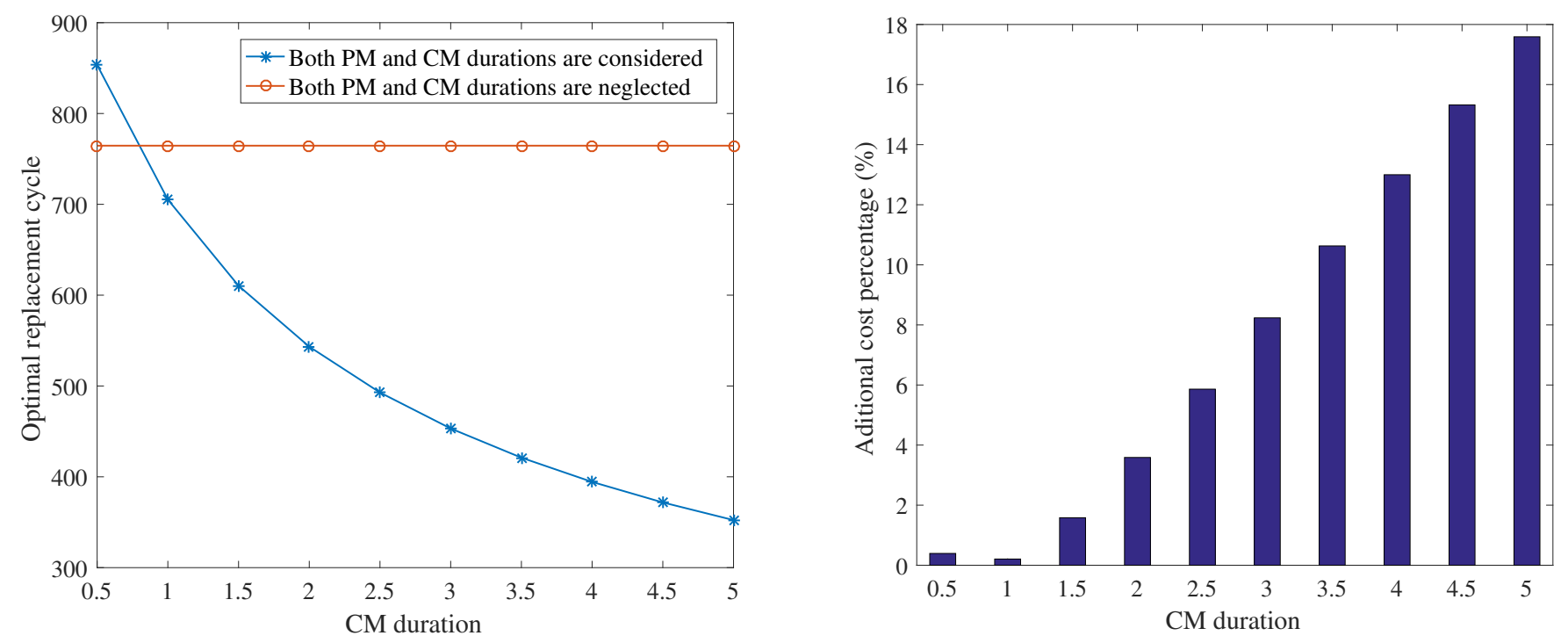

Figure 6: Optimal replacement cycle (a) and aditional cost percentage (b) versus different values of CM duration

values of the CM duration. From the Figure 6a, we can see that in most cases there exists always the difference between the optimal replacement cycle value obtained in non-negligible maintenance duration case and that obtained in negligible maintenance duration one. The difference leads to an additional maintenance cost when the maintenance duration are not considered during the maintenance planning. In this analysis, the additional cost can increase until $17.80 \%$ of the maintenance cost rate of the component (see Figure $6 \mathrm{~b}$ ). The additional cost percentage plotted in Figure 6b is calculated as

$$
\Delta C R_{i}=\frac{C R_{i}\left(x_{i}^{\overline{P C}}\right)-C R_{i}\left(x_{i}^{P C}\right)}{C R_{i}\left(x_{i}^{P C}\right)} \cdot 100
$$

$\Delta C R_{i}$ represents the additional maintenance cost that we have to paid if we do not take into account the CM duration during the maintenance planning. From the above analysis, we should underline also that the impacts of CM duration on the individual maintenance plan and cost are significant and can not be neglected even when it is very small.

In this step, the sensitivity analysis for different structure types is not carried out because the interactions among components are not considered.

\subsection{Tentative maintenance planning}

The aim of this step is to determine the tentative PM dates within a short-term planning horizon with taking into account the interactions among components. To avoid the complexity in determining the tentative maintenance dates based on the optimal age-based replacement cycle $\left(x_{i}^{P C}\right)$, the calendar-based $\left(T_{i}\right)$ one was proposed. The calendar-based replacement cycle, the tentative PM dates for different cases, and the age of components at their replacement time are calculated and reported in Table 5. The planning horizon is then selected as $P H=\left[0, t_{4^{1}}+\omega^{4 p}\right]=[0,486.2]$.

Graphical representation of the tentative maintenance plans in the three different cases is shown in Figure 7.

Table 5 and Figure 7 confirm clearly that the consideration of maintenance duration makes the tentative maintenance dates change. While the age of components at their maintenance dates is equal to their replacement 
Table 5: Tentative maintenance planning.

\begin{tabular}{lllllll}
\hline Components & 1 & 2 & 3 & 4 & 5 & 6 \\
\hline$T_{i}$ & 466.2 & 508.5 & 653.8 & 492.2 & 480.9 & 529.3 \\
$t_{i^{1}}^{P C}$ & 888.4 & 618.4 & 750.5 & 780.7 & 714.6 & 809.3 \\
$t_{i^{1}}^{P \bar{C}}$ & 1078.0 & 683.1 & 816.2 & 862.4 & 1086 & 991.6 \\
$t_{i^{1}}^{P C}$ & 366.2 & 358.5 & 398.8 & 482.2 & 430.9 & 429.3 \\
$x_{i}\left(t_{i^{1}}^{P C}\right)$ & 453.1 & 484.8 & 627.6 & 461.8 & 461.0 & 513.0 \\
\hline
\end{tabular}

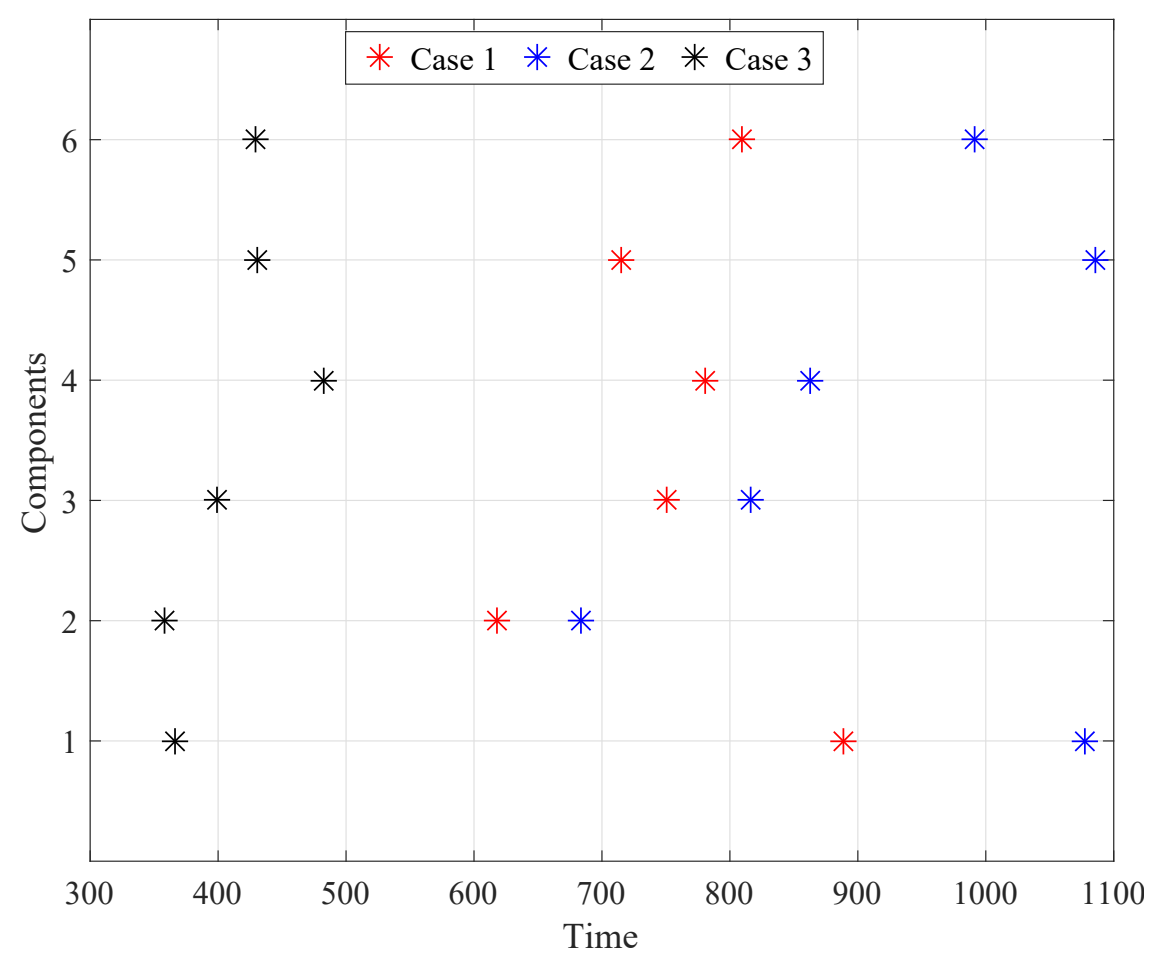

Figure 7: Tentative maintenance plans vs different considered cases

cycles when both PM and CM duration are neglected or when only PM duration is taken into account, the age of components at their PM dates when CM duration is taken into account is not equal to the replacement cycle, i.e. $x_{i}\left(t_{i^{1}}^{P C}\right) \neq T_{i}$. The use of calendar-based replacement threshold can facilitate the tentative maintenance planning, however it also leads to many difficulties in determining the component's age, especially when both the system structure and maintenance duration are considered. The age of one component depends on the maintenance duration of the other ones in the system. To solve the problem, the proposed process: component's age assessment and component's criticality determination were then used.

To study the impacts of system structure and maintenance duration on the age evolution of a component, component 3 was selected and its age evolution is plotted in Figure 8 for the different cases. Note that the increase of the component age when both PM and CM duration are neglected is equal to that of calendar time. In the figure, the taking into account of only PM duration has a very small impact on the evolution of the component's age. In fact, the age evolution of component 3 is affected only at some determined times when the other components in the system are preventively maintained. Otherwise, the difference between the evolution of 


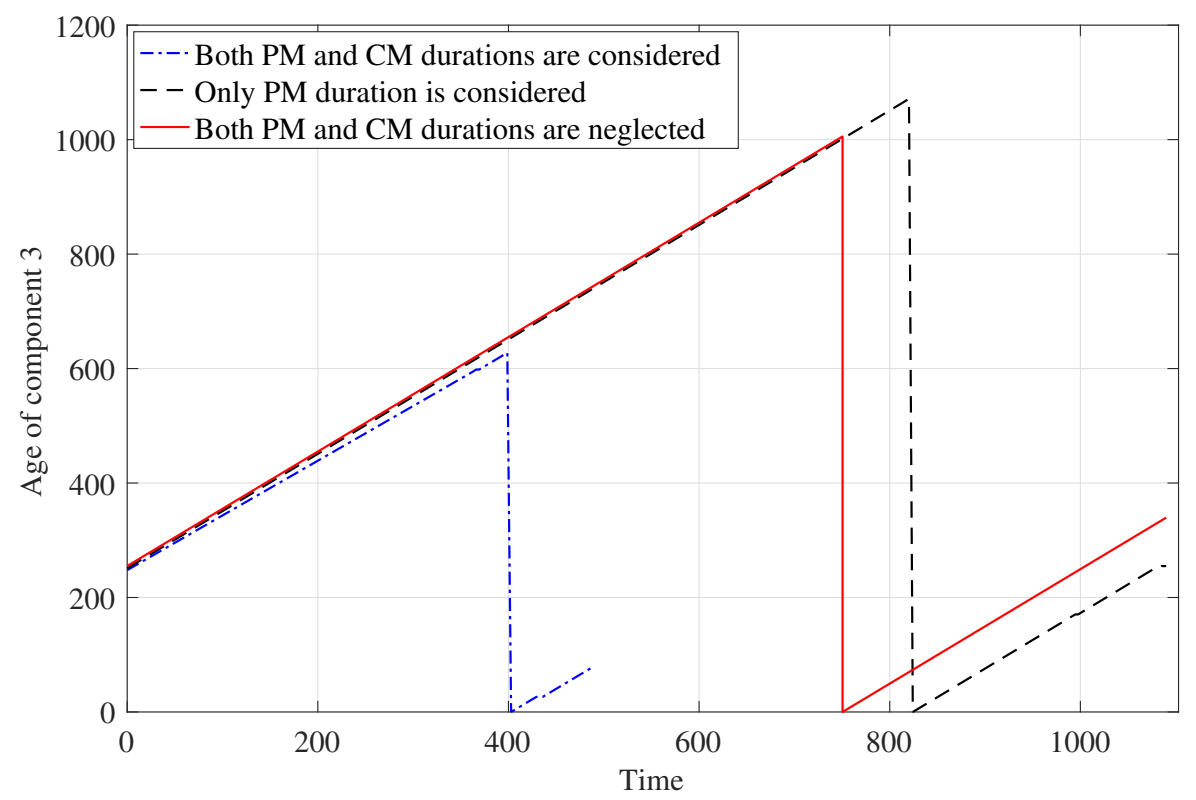

Figure 8: Age evolution of component 3 with different maintenance duration assumptions

the component age and that of calendar time always exists when the CM duration is taken into consideration.

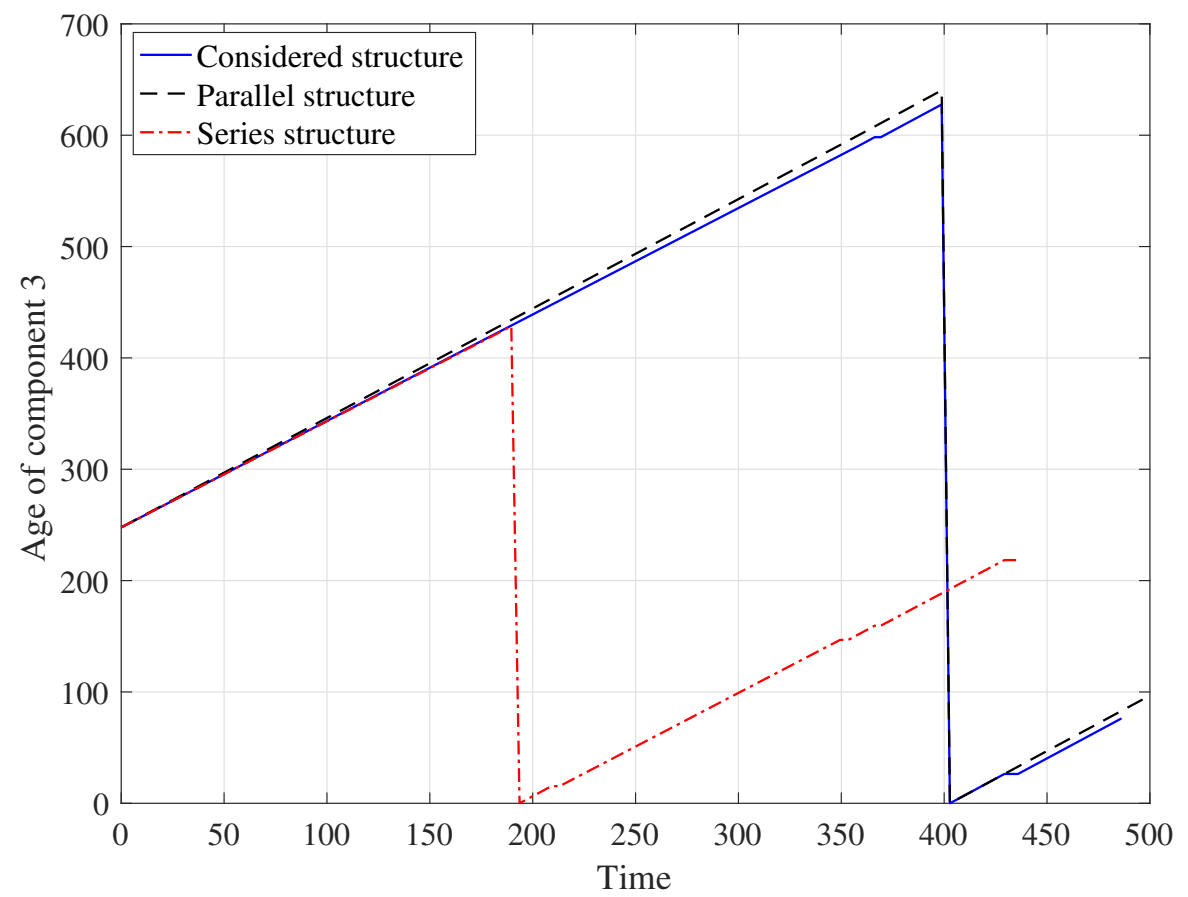

Figure 9: Age evolution of component 3 with different system structure types

It should be noted that the system structure is also an important factor that affects the age's evolution of the component. To make it clear, age evolution of component 3 with taking into consideration both PM and CM durations is plotted in Figure 9 for the different structure types. From the Figure, we can see that for parallel structure, the age evolution and calendar time evolution are similar, in spite of non-negligible maintenance duration. Contrarily, they are not equal in cases of the other structure types, especially series type since the 
operation of the component is affected by all maintenance activities (PM and CM) of all other components in the system.

\subsection{Grouping maintenance planning}

In this subsection, the tentative PM dates obtained in the previous step are grouped in order to reduce the maintenance cost. The optimal grouping solution containing the optimal grouping structure and the optimal grouping execution dates are found by using different optimization tools. Given there are only 6 PM activities within the planning horizon, the finding of the optimal grouping structure can be done by the use of the exhaustive search method who examines all 203 possible grouping structures. Otherwise, under the impacts of CM duration and system structure, the optimal execution times of groups can not be determined separately. The Genetic Algorithm tool box of Matlab is used in this paper to jointly find the optimal execution times of groups. To partially reduce the uncertainty in the results provided by GA, it is run five times and then the best result is recorded. Similar to the above analysis of the individual maintenance optimization, the grouping optimization is also analyzed for the different cases of maintenance duration assumptions and system structure types.

Table 6: Optimal grouping solutions vs different maintenance duration assumptions.

\begin{tabular}{|c|c|c|c|c|c|c|c|}
\hline Assumptions & $\begin{array}{l}\text { Optimization } \\
\text { of } t_{G^{k}}\end{array}$ & $G S^{*}$ & $t_{G^{k}}^{*}$ & $E P$ & $\begin{array}{c}\text { Saving } \\
\text { percentage }\end{array}$ & $E P^{\prime}$ & $\begin{array}{l}\text { Saving } \\
\text { reduction }\end{array}$ \\
\hline $\begin{array}{c}\text { Case 1: Both PM and CM } \\
\text { duration are neglected }\end{array}$ & $\begin{array}{c}\text { Joint \& separate } \\
\text { optimizations }\end{array}$ & $\begin{array}{c}G^{1}=\{2\} \\
G^{2}=\{1,3,4,5,6\}\end{array}$ & $\begin{array}{l}618.4 \\
---- \\
784.5 \\
\end{array}$ & 16.92 & $0.35 \%$ & 139.96 & $34.49 \%$ \\
\hline $\begin{array}{c}\text { Case 2: Only PM duration } \\
\text { is considered }\end{array}$ & $\begin{array}{c}\text { Joint \& separate } \\
\text { optimizations }\end{array}$ & $\begin{array}{c}G^{1}=\{2\} \\
G^{2}=\{3\} \\
G^{3}=\{4\} \\
G^{4}=\{1,5,6\}\end{array}$ & $\begin{array}{l}687.1 \\
---- \\
820.2 \\
---- \\
866.4 \\
---- \\
1049.4 \\
\end{array}$ & 187.33 & $2.73 \%$ & 152.29 & $28.71 \%$ \\
\hline \multirow{2}{*}{$\begin{array}{c}\text { Case 3: Both PM and CM } \\
\text { duration are considered }\end{array}$} & $\begin{array}{c}\text { Separate } \\
\text { optimization }\end{array}$ & $\begin{array}{c}G^{1}=\{1,3,5,6\} \\
-G^{2}=\{2,4\}\end{array}$ & $\begin{array}{c}397.7 \\
---- \\
412.9\end{array}$ & 192.11 & $3.16 \%$ & 192.11 & $10.07 \%$ \\
\hline & $\begin{array}{c}\text { Joint } \\
\text { optimization }\end{array}$ & $\begin{array}{c}G^{1}=\{1,3,5,6\} \\
G^{2}=\{2,4\}\end{array}$ & $\begin{array}{l}445.9 \\
---- \\
450.9\end{array}$ & 213.63 & $3.52 \%$ & 213.63 & $0 \%$ \\
\hline
\end{tabular}

Table 6 and Figure 10 represent the grouping results for the different maintenance duration assumptions. With respect to a specific maintenance duration assumption, the optimal execution dates of groups are found by two different ways: (a) separately based on $\Delta H_{G^{k}}^{1}$; (b) jointly by using Genetic algorithm. From the obtained results, we can conclude that the joint optimization is necessary to guarantee the grouping performance in case that both PM and CM duration are considered. In other words, the optimization process takes more computing time and more complex when CM duration is considered. Given the challenge in grouping optimization, the taking into account of the maintenance duration is of interest to improve the grouping performance. Indeed, the grouping maintenance can save $2.73 \%$ and $3.52 \%$ of the total individual maintenance cost in case 2 and case 3 respectively. Otherwise, in case 1 , the saving percentage is only $0.35 \%$. The grouping maintenance is then a good choice for the applications where the maintenance duration are non-negligible. In addition, imagine that 


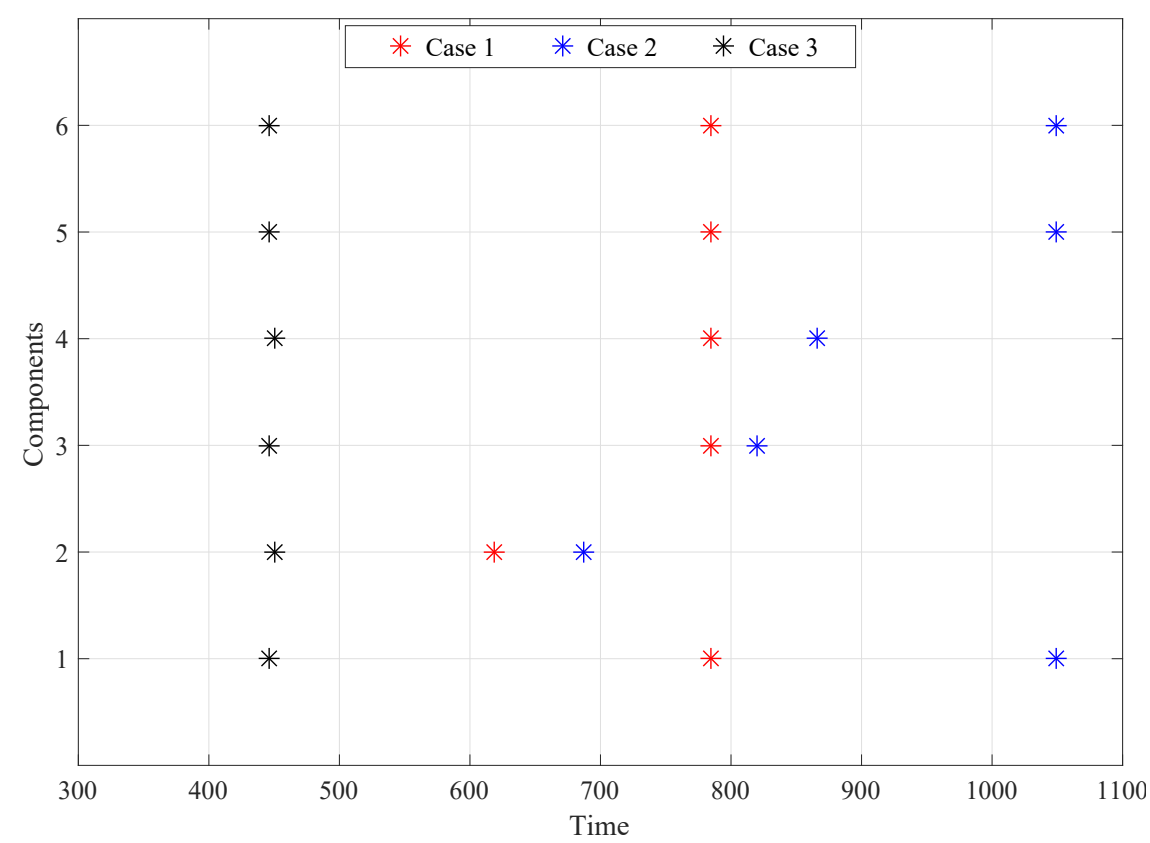

Figure 10: Grouping maintenance plans vs different considered cases

the optimal grouping solutions obtained in cases 1 and 2 are now used for the maintenance of the considered system with non-negligible PM and CM duration, the maintenance cost saving is reduced around $34.49 \%$ and $28.71 \%$ when compared to the case when the optimal grouping solution obtained in case 3 is applied.

To verify the grouping performance in different system structure and maintenance duration settings, a number of analysis have been realized. Figure 11 and Table 7 represent the results obtained from the sensitivity analysis of grouping profit and grouping structure to different values of PM duration from 1 to 8 and to different structure types.

In Figure 11, the grouping economic profit increases very quickly as the PM duration increases if the structure is series or complex one, otherwise, it is nearly constant in case of the parallel structure. The taking into account PM duration in the grouping optimization process is therefore not realy necessary in case of parallel structure, otherwise it is very important and can not be neglected in cases of series and complex structures.

Table 7: Optimal grouping structures vs different structure types.

\begin{tabular}{|c|l|l|}
\hline Structure types & PM duration & Optimal grouping structure \\
\hline \hline \multirow{3}{*}{ Complex structure } & $\omega^{i p}=[1,3]$ & $G^{1}=\{4\}, G^{2}=\{1,2,3,5,6\}$ \\
\cline { 2 - 3 } & $\omega^{i p} \in(3,8]$ & $G^{1}=\{3,4\}, G^{2}=\{1,2,5,6\}$ \\
\hline \multirow{3}{*}{ Parallel structure } & $\omega^{i p}=[1,2]$ & $G^{1}=\{2,3\}, G^{2}=\{4,5\}, G^{3}=\{1,6\}$ \\
\cline { 2 - 3 } & $\omega^{i p} \in(2,8]$ & $G^{1}=\{2,3\}, G^{2}=\{4\}, G^{3}=\{1,5,6\}$ \\
\hline \multirow{2}{*}{ Series structure } & $\omega^{i c} \in[1,3]$ & $G^{1}=\{2,3\}, G^{2}=\{1,4,5,6\}$ \\
\cline { 2 - 3 } & $\omega^{i p} \in(3,8]$ & $G^{1}=\{1,2,3,4,5,6\}$ \\
\hline
\end{tabular}

Table 7 reports the changes of the optimal grouping structure when PM duration increases from 1 to 8 for different structure types. The optimal grouping structure is changed for different PM duration intervals. The 


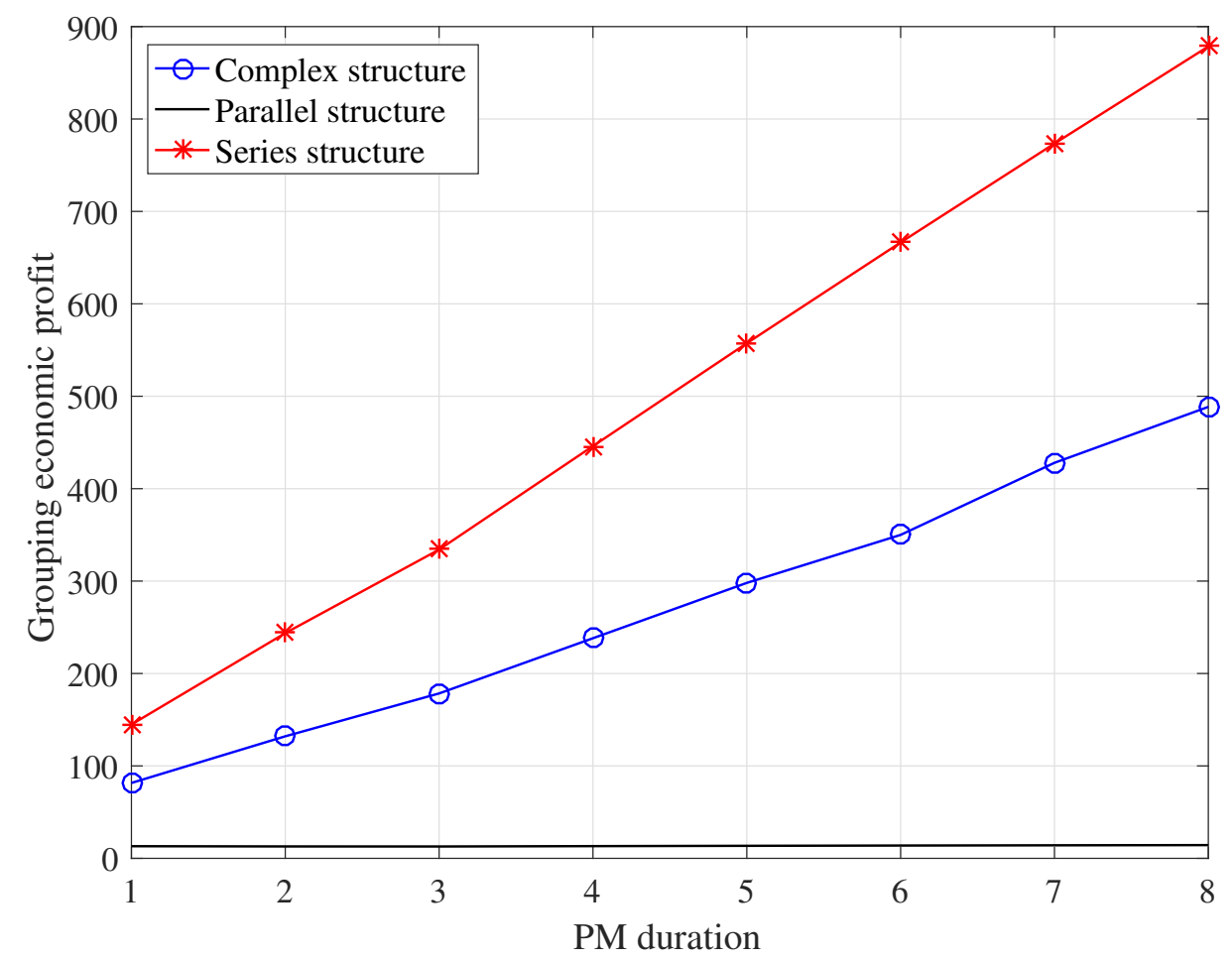

Figure 11: Grouping economic profit as function of PM duration

components are grouped more frequent to save the downtime and shutdown costs in the series and complex structures.

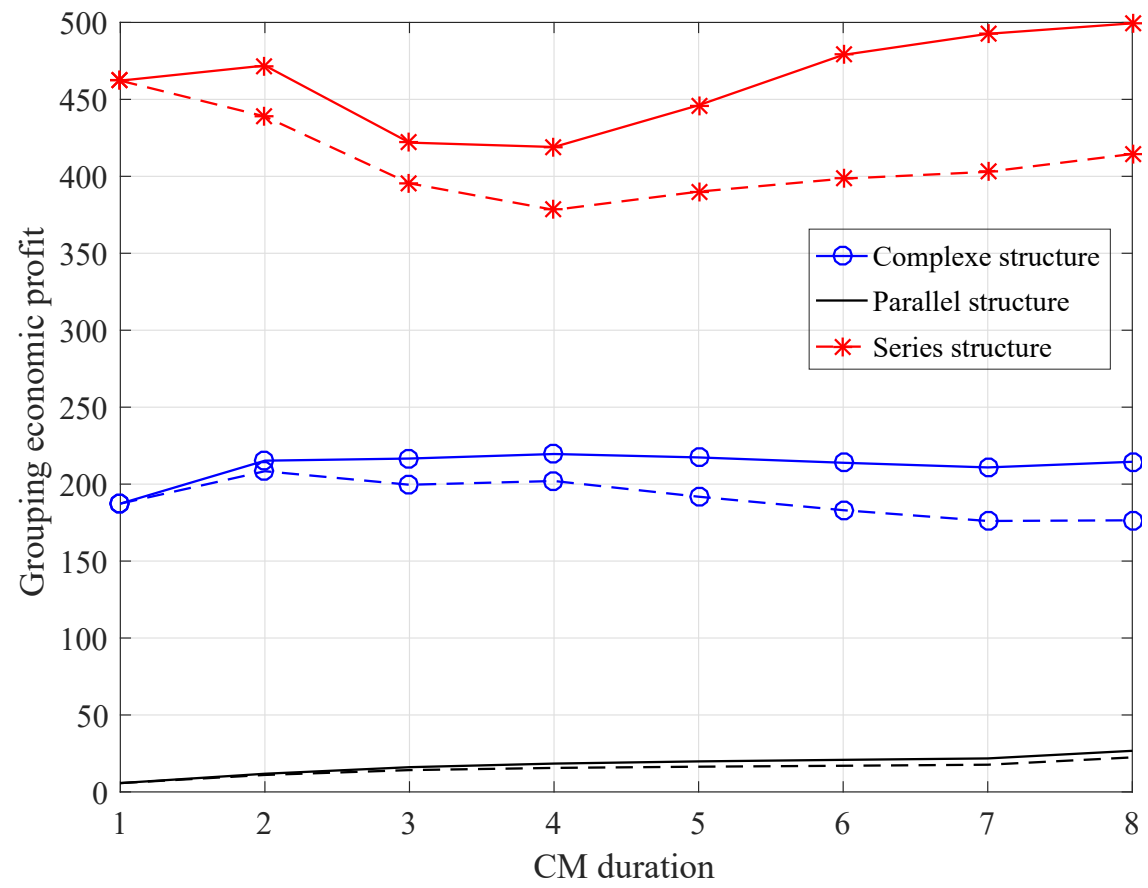

Figure 12: Grouping economic profit as a function CM duration

The same sensitivity analysis of grouping maintenance are carried out, in which the PM duration is fixed, while the CM duration increases from 1 to 8. The obtained results are shown in Figure 12 and Table 8. From the Figure 12, we can see that differently to the PM duration case, the grouping performance is not much 
sensitive and not proportional to the CM duration value. In addition, the dash lines in the figure represent the cases where the execution times of groups are separately optimized. The bigger the CM duration value is, the higher distance between the dash lines and corresponding continue lines is. The grouping optimization process is strongly affected by the CM duration. Finally, it should be noticed that for a parallel structure, both PM and CM duration has small impacts on the grouping performance.

Table 8: Optimal grouping structures vs different structure types.

\begin{tabular}{|c|l|l|}
\hline Structure types & CM duration & Optimal grouping structure \\
\hline \hline \multirow{3}{*}{ Complex structure } & $\omega^{i c} \in[1,2]$ & $G^{1}=\{3,4\}, G^{2}=\{1,2,5,6\}$ \\
\cline { 2 - 3 } & $\omega^{i c} \in(2,4]$ & $G^{1}=\{2,4\}, G^{2}=\{1,3,5,6\}$ \\
\cline { 2 - 3 } & $\omega^{i c} \in(4,8]$ & $G^{1}=\{4\}, G^{2}=\{1,2,3,5,6\}$ \\
\hline Parallel structure & $\omega^{i c} \in[1,8]$ & $G^{1}=\{2,3\}, G^{2}=\{4\}, G^{3}=\{1,5,6\}$ \\
\hline \multirow{2}{*}{ Series structure } & $\omega^{i c} \in[1,2]$ & $G^{1}=\{1,2,3,4,5,6\}$ \\
\cline { 2 - 3 } & $\omega^{i c} \in(2,8]$ & $G^{1}=\{2,3\}, G^{2}=\{1,4,5,6\}$ \\
\hline
\end{tabular}

Table 8 reports the changes of the optimal grouping structure when the CM duration varies from 1 to 8 . The taking into account of CM duration leads to changes in the optimal grouping structure. For series structure, when CM duration is high, the grouping maintenance will lead to high CM penalty cost, the grouping structure therefore contains more groups than the case when CM duration is small. For parallel structure, the grouping structure seems to be independent from the CM duration.

\section{Conclusion}

In this paper, a dynamic grouping approach is developed for the maintenance planning of the complex structure systems with consideration of both preventive and corrective maintenance duration. A new maintenance cost structure and grouping maintenance model allowing taking into account the maintenance duration and the dynamic system structure as well as economic dependence between components is firstly proposed.

Secondly, despite the complexity of the considered grouping model and grouping optimization process, the consideration of the maintenance duration and system redundancy is integrated by two proposed analytical methods which help to avoid the problem of computational time, and make the dynamic update of the grouping maintenance plan possible in real applications. The performance of the proposed grouping strategy and the necessity of the consideration of maintenance duration and system structure are highlighted through an example of a six-component system.

Moreover, through the paper, the impacts of maintenance duration and system structure on the individual optimization and grouping maintenance are studied both theoretically and experimentally. The results obtained from different sensitivity analyses show that PM duration has a strongly impact on the grouping performance (economic profit). Otherwise, the grouping accuracy depends considerably on CM duration. From a system structure point of view, the grouping maintenance of series structure is the most sensitive to the PM and CM duration. In real applications, the grouping maintenance is strongly recommended for the maintenance planning of series systems with consideration of PM duration since it can help to reduce significantly the maintenance cost. 
For the parallel structure systems, the grouping maintenance and the taking into account of the maintenance duration on the grouping process are not interesting since the operational dependencies among components of such systems are very weak. In addition, the results obtained in case of redundant structure systems are always bounded by that of series and parallel ones. The results are reasonable since the parallel and series structures are two extreme cases of the redundant level. The research helps to orientate the use of the grouping maintenance in real applications.

This paper is the development of our research in the framework of maintenance modelling and optimization in presence of maintenance duration presented partially in [36]. Our future research works focus on the investigation of the proposed grouping maintenance approach with consideration of random maintenance duration models, stochastic dependence and logistic supports specifications/constraints. An other important research direction could be the application of the proposed approach to a real case study with real failure and maintenance cost data.

\section{A Individual maintenance optimization: optimality analysis}

The optimal value of $x_{i}^{0}$, denoted $x_{i}^{*}$, which minimizes the above long-term maintenance cost rate, can be determined by solving Equation 8.

$$
\left.\frac{d C R_{i}}{d x_{i}}\right|_{x_{i}^{0}=x_{i}^{*}}=0 \Leftrightarrow C^{i c} \cdot\left(\beta_{i}-1\right) \cdot\left(x_{i}^{*}\right)^{\beta_{i}}+\left(C^{i c} \cdot \omega^{i p}-C^{i p} \cdot \omega^{i c}\right) \cdot \beta_{i} \cdot\left(x_{i}^{*}\right)^{\beta_{i}-1}-C^{i p} \cdot \lambda_{i}^{\beta_{i}}=0
$$

\section{A.1 Only PM duration is considered}

When only PM duration is considered, CM duration is neglected $\omega^{i c}=0$. Equation 8 becomes

$$
f\left(x_{i}^{P \bar{C}}\right)=C_{i n}^{i c} \cdot\left(\beta_{i}-1\right) \cdot\left(x_{i}^{P \bar{C}}\right)^{\beta_{i}}+C_{i n}^{i c} \cdot \omega^{i p} \cdot \beta_{i} \cdot\left(x_{i}^{P \bar{C}}\right)^{\beta_{i}-1}-C^{i p} \cdot \lambda_{i}^{\beta_{i}}=0
$$

We have

$$
f(0)=-C^{i p} \cdot \lambda_{i}^{\beta_{i}}<0
$$

and

$$
f\left(a_{1}\right)=C_{i n}^{i c} \cdot \omega^{i p} \cdot \beta_{i} \cdot\left(a_{1}\right)^{\beta_{i}-1} \geq 0
$$

where

$$
a_{1}=\lambda_{i} \sqrt[\beta_{i}]{\frac{C^{i p}}{C_{i n}^{i c} \cdot\left(\beta_{i}-1\right)}}
$$

In addition,

$$
f^{\prime}\left(x_{i}^{P \bar{C}}\right)=C_{i n}^{i c} \cdot\left(\beta_{i}-1\right) \cdot \beta_{i} \cdot\left(x_{i}^{P \bar{C}}\right)^{\beta_{i}-1}+C_{i n}^{i c} \cdot \beta_{i} \cdot \omega^{i p} \cdot\left(\beta_{i}-1\right) \cdot\left(x_{i}^{P \bar{C}}\right)^{\beta_{i}-2}>0, \forall \beta_{i}>1, \forall x_{i}^{P \bar{C}}>0 ;
$$

From Equations 36, 37, 38, we can conclude that Equation 8 has only one solution $x_{i}^{P \bar{C}}$ and $x_{i}^{P \bar{C}} \in\left(0, a_{1}\right]$. Moreover, it is not difficult to show that the second derivative of the long-term maintenance cost rate $C R_{i}$ at $x_{i}^{P \bar{C}}$ is positive.

$$
C R_{i}^{\prime \prime}\left(x_{i}^{P \bar{C}}\right)=\frac{C_{i n}^{i c} \cdot\left(\beta_{i}-1\right) \cdot \beta_{i} \cdot\left(x_{i}^{P \bar{C}}\right)^{\beta_{i}-1}+C_{i n}^{i c} \cdot\left(\beta_{i}-1\right) \cdot \beta_{i} \cdot \omega^{i p} \cdot\left(C_{i n}^{i c}\right)^{\beta_{i}-2}}{\left(C_{i n}^{i c}+\omega^{i p}\right)^{2}}>0
$$

Consequently, the optimal replacement cycle $x_{i}^{P \bar{C}}$ exists and is unique in open interval $\left(0, a_{1}\right]$. 


\section{A.2 Both PM and CM duration are considered}

When both PM and CM duration are considered $\omega^{i p} \neq 0$, and $\omega^{i c} \neq 0$, the optimal replacement cycle $x_{i}^{P C}$ is found by solving Equation 8.

$$
g\left(x_{i}^{P C}\right)=C^{i c} \cdot\left(\beta_{i}-1\right) \cdot\left(x_{i}^{P C}\right)^{\beta_{i}}+\left(C^{i c} \cdot \omega^{i p}-C^{i p} \cdot \omega^{i c}\right) \cdot \beta_{i} \cdot\left(x_{i}^{P C}\right)^{\beta_{i}-1}-C^{i p} \cdot \lambda_{i}^{\beta_{i}}=0
$$

We consider the following values of $g\left(x_{i}^{P C}\right)$ :

$$
\begin{gathered}
g(0)=-C^{i p} \cdot \lambda_{i}^{\beta_{i}}<0 \\
g\left(a_{2}\right)=\left(C^{i c} \omega^{i p}-C^{i p} \cdot \omega^{i c}\right) \cdot \beta_{i} \cdot\left(a_{2}\right)^{\beta_{i}-1}
\end{gathered}
$$

where

$$
\begin{gathered}
a_{2}=\sqrt[\beta_{i}]{\frac{C^{i p} \cdot \lambda_{i}^{\beta_{i}}}{C^{i c} \cdot\left(\beta_{i}-1\right)}} \\
g(b)=C^{i c} \cdot\left(\beta_{i}-1\right) \cdot(b)^{\beta_{i}}>0
\end{gathered}
$$

where

$$
b=\sqrt[\beta_{i}-1]{\frac{C^{i p} \cdot \lambda_{i}^{\beta_{i}}}{\beta_{i} \cdot\left(C^{i c} \omega^{i p}-C^{i p} \cdot \omega^{i c}\right)}}
$$

To ensure that the long-term maintenance cost rate is minimal at $x_{i}^{P C}$, its second derivative $C R_{i}^{\prime \prime}$ must be bigger than 0 . We have

$$
\begin{gathered}
C R_{i}^{\prime \prime}\left(x_{i}^{P C}\right)=\frac{C^{i c} \cdot\left(\beta_{i}-1\right) \cdot \beta_{i} \cdot\left(x_{i}^{P C}\right)^{\beta_{i}-1}+\left(C^{i c} \omega^{i p}-C^{i p} \cdot \omega^{i c}\right) \cdot\left(\beta_{i}-1\right) \cdot \beta_{i} \cdot\left(x_{i}^{P C}\right)^{\beta_{i}-2}}{\left(x_{i}^{P C}+\omega^{i c} \cdot\left(\frac{x_{i}^{P C}}{\lambda_{i}}\right)^{\beta_{i}}+\omega^{i p}\right)^{2}}>0 \\
\Leftrightarrow x_{i}^{P C}>c=\frac{C^{i p} \cdot \omega^{i c}-C^{i c} \omega^{i p}}{C^{i c}}
\end{gathered}
$$

and

$$
g(c)=-\left(C^{i p} \cdot \omega^{i c}-C^{i c} \omega^{i p}\right)^{\beta_{i}}-C^{i p} \cdot \lambda_{i}^{\beta_{i}}
$$

Finally, two following cases are distinguished:

- if If $\frac{C^{i c}}{C^{i p}}>\frac{\omega^{i c}}{\omega^{i p}}$, we then have $g\left(a_{2}\right)>0, g(b)>0$, and $g(0)<0$. The optimal replacement cycle $x_{i}^{P C}$ exists and is unique in interval $\left(0, \min \left(a_{2}, b\right)\right)$.

- if If $\frac{C^{i c}}{C^{i p}} \leq \frac{\omega^{i c}}{\omega^{i p}}$, then $g\left(a_{2}\right) \leq 0, g(c)<0$, and $g(+\infty)>0$. As a consequence, $x_{i}^{P C}$ exists and is unique in interval $\left[\max \left(a_{2}, c\right),+\infty\right)$.

\section{References}

[1] R. Barlow and L. Hunter. Optimum preventive maintenance policies. Operations research, 8(1):90-100, 1960.

[2] H. Pham and H. Wang. Imperfect maintenance. European journal of operational research, 94(3):425-438, 1996.

[3] T. Nakagawa. A summary of periodic replacement with minimal repair at failure. Journal of the Operations Research Society of Japan, 24(3):213-227, 1981.

[4] H.W. Block, N.A. Langberg, and T.H. Savits. Repair replacement policies. Journal of Applied Probability, 30(1):194-206, 1993. 
[5] R.P. Nicolai and R. Dekker. Optimal maintenance of multi-component systems: a review. Complex system maintenance handbook, pages 263-286, 2008.

[6] H. Wang. A survey of maintenance policies of deteriorating systems. European Journal of Operational Research, 139(3):469-489, 2002.

[7] S. Alaswad and Y. Xiang. A review on condition-based maintenance optimization models for stochastically deteriorating system. Reliability Engineering \& System Safety, 157:54-63, 2017.

[8] R. Ahmad and S. Kamaruddin. An overview of time-based and condition-based maintenance in industrial application. Computers \& Industrial Engineering, 63(1):135-149, 2012.

[9] R. Dekker, R.E. Wildeman, and F.A. van der Duyn Schouten. A review of multi-component maintenance models with economic dependence. Mathematical Methods of Operations Research, 45(3):411-435, 1997.

[10] T. Nowakowski and S. Werbińka. On problems of multicomponent system maintenance modelling. International Journal of Automation and Computing, 6(4):364-378, 2009.

[11] E. Deloux and L. Dieulle. Condition-based maintenance policies for multi-component systems with stochastic, structural and economic dependencies. IMA International Conference on Modelling in Industrial Maintenance and Reliability, Oxford, UK, 2014.

[12] M.C.O. Keizer, S.D.P. Flapper, and R.H. Teunter. Condition-based maintenance policies for systems with multiple dependent components: A review. European Journal of Operational Research, 261(2):405-420, 2017.

[13] H.C. Vu, P. Do, A. Barros, and C. Bérenguer. Maintenance planning and dynamic grouping for multicomponent systems with positive and negative economic dependencies. IMA Journal of Management Mathematics, 26(2):145-170, 2015.

[14] K.A. Nguyen, P. Do, and A. Grall. Multi-level predictive maintenance for multi-component systems. Reliability Engineering \& System Safety, 144:83-94, 2015.

[15] H. Pham and H. Wang. Optimal $(\tau, \mathrm{t})$ opportunistic maintenance of ak-out-of-n: G system with imperfect pm and partial failure. Naval Research Logistics (NRL), 47(3):223-239, 2000.

[16] X. Zhou, L. Xi, and J. Lee. Opportunistic preventive maintenance scheduling for a multi-unit series system based on dynamic programming. International Journal of Production Economics, 118(2):361-366, 2009.

[17] H. Ab-Samat and S. Kamaruddin. Opportunistic maintenance (om) as a new advancement in maintenance approaches: A review. Journal of Quality in Maintenance Engineering, 20(2):98-121, 2014.

[18] N. Chalabi, M. Dahane, B. Beldjilali, and A. Neki. Optimisation of preventive maintenance grouping strategy for multi-component series systems: Particle swarm based approach. Computers \& Industrial Engineering, 102:440-451, 2016.

[19] T. Nakagawa and S. Mizutani. A summary of maintenance policies for a finite interval. Reliability Engineering \& System Safety, 94(1):89-96, 2009.

[20] J. Wu, T.S.A. Ng, M. Xie, and H.Z. Huang. Analysis of maintenance policies for finite life-cycle multi-state systems. Computers \& Industrial Engineering, 59(4):638-646, 2010.

[21] J.I. Aizpurua, V.M. Catterson, Y. Papadopoulos, F. Chiacchio, and D. D'Urso. Supporting group maintenance through prognostics-enhanced dynamic dependability prediction. Reliability Engineering \& System Safety, 2017. 
[22] S.K. Goyal and M.I. Kusy. Determining economic maintenance frequency for a family of machines. Journal of the Operational Research Society, 36(12):1125-1128, 1985.

[23] G. van Dijkhuizen and A. van Harten. Optimal clustering of frequency-constrained maintenance jobs with shared set-ups. European Journal of Operational Research, 99(3):552-564, 1997.

[24] R.E. Wildeman, R. Dekker, and A. Smit. A dynamic policy for grouping maintenance activities. European Journal of Operational Research, 99(3):530-551, 1997.

[25] K. Bouvard, S. Artus, C. Bérenguer, and V. Cocquempot. Condition-based dynamic maintenance operations planning \& grouping. application to commercial heavy vehicles. Reliability Engineering \& System Safety, 96(6):601-610, 2011.

[26] A. V. Horenbeek and L. Pintelon. A dynamic predictive maintenance policy for complex multi-component systems. Reliability Engineering and System Safety, 120:39-50, 2013.

[27] P. Okoh. Maintenance grouping optimization for the management of risk in offshore riser system. Process Safety and Environmental Protection, 98:33-39, 2015.

[28] M. Sheikhalishahi, A. Azadeh, and L. Pintelon. Dynamic maintenance planning approach by considering grouping strategy and human factors. Process Safety and Environmental Protection, 107:289-298, 2017.

[29] H. Li, L. Dieulle, and E. Deloux. Condition-based maintenance policies for multi-component systems with lévy copulas dependence. In Safety and Reliability: Methodology and Applications, pages 1281 -1288. Taylor \& Francis Group, London, 2014.

[30] H.C. Vu, P. Do, A. Barros, and C. Bérenguer. Maintenance grouping strategy for multi-component systems with dynamic contexts. Reliability Engineering \& System Safety, 132:233-249, 2014.

[31] P. Do, A. Barros, K. Bérenguer, C. Bouvard, and F. Brissaud. Dynamic grouping maintenance with time limited opportunities. Reliability Engineering \& System Safety, 120:51-59, 2013.

[32] P. Do, H.C. Vu, A. Barros, and C. Bérenguer. Maintenance grouping for multi-component systems with availability constraints and limited maintenance teams. Reliability Engineering \& System Safety, 142:56-67, 2015.

[33] R.E. Wildeman and R. Dekker. Dynamic influences in multi-component maintenance. Quality and reliability engineering international, 13(4):199-207, 1997.

[34] M. Rausand and H. Arnljot. System reliability theory: models, statistical methods, and applications, volume 396. John Wiley \& Sons, 2004.

[35] Michael F Doherty and Michael F Malone. Conceptual design of distillation systems. McGraw-Hill Science/Engineering/Math, 2001.

[36] H.C. Vu, P. Do, A. Barros, and M.A. Lundteigen. A dynamic grouping model for the maintenance planning of complex structure systems with consideration of maintenance durations. In 1st International Conference on Applied Mathematics in Engineering and Reliability, ICAMER 2016, number ISBN 978-161-138-02928-6, pages 73-80. CRC Press/Balkema, Taylor \& Francis Group, 2016. 